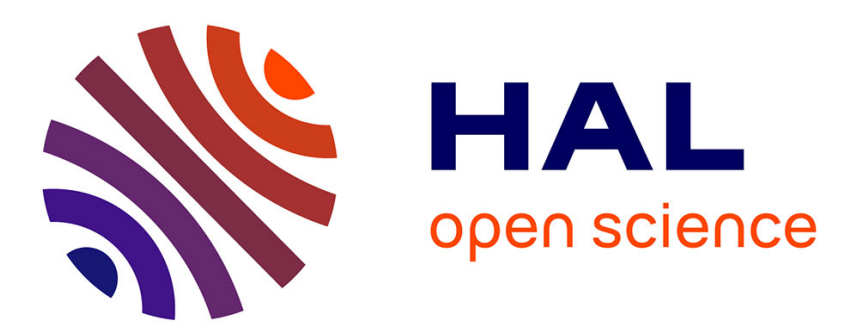

\title{
Evidential Matrix Metrics as Distances Between Meta-Data Dependent Bodies of Evidence
} Mehena Loudahi, John Klein, Jean-Marc Vannobel, Olivier Colot

\section{To cite this version:}

Mehena Loudahi, John Klein, Jean-Marc Vannobel, Olivier Colot. Evidential Matrix Metrics as Distances Between Meta-Data Dependent Bodies of Evidence. IEEE transactions on systems, man, and cybernetics, 2016, 46 (1), pp.109 - 122. 10.1109/TCYB.2015.2395877 . hal-01115637

\section{HAL Id: hal-01115637 \\ https://hal.science/hal-01115637}

Submitted on 11 Feb 2015

HAL is a multi-disciplinary open access archive for the deposit and dissemination of scientific research documents, whether they are published or not. The documents may come from teaching and research institutions in France or abroad, or from public or private research centers.
L'archive ouverte pluridisciplinaire HAL, est destinée au dépôt et à la diffusion de documents scientifiques de niveau recherche, publiés ou non, émanant des établissements d'enseignement et de recherche français ou étrangers, des laboratoires publics ou privés. 


\title{
Evidential matrix metrics as distances between meta-data dependent bodies of evidence
}

\author{
Mehena Loudahi, John Klein, Jean-Marc Vannobel and Olivier Colot *†
}

\begin{abstract}
As part of the theory of belief functions, we address the problem of appraising the similarity between bodies of evidence in a relevant way using metrics. Such metrics are called evidential distances and must be computed from mathematical objects depicting the information inside bodies of evidence. Specialization matrices are such objects and, therefore, an evidential distance can be obtained by computing the norm of the difference of these matrices. Any matrix norm can be thus used to define a full metric.

In this article, we show that other matrices can be used to obtain new evidential distances. These are the $\alpha$-specialization and $\alpha$-generalization matrices and are closely related to the $\alpha$-junctive combination rules. We prove that any $L^{1}$ norm-based distance thus defined is consistent with its corresponding $\alpha$-junction. If $\alpha>0$, these distances have in addition relevant variations induced by the poset structure of the belief function domain. Furthermore, $\alpha$-junctions are meta-data dependent combination rules. The meta-data involved in $\alpha$-junctions deals with the truthfulness of information sources. Consequently, the behavior of such evidential distances is analyzed in situations involving uncertain or partial metaknowledge about information source truthfulness.
\end{abstract}

Index terms - Theory of belief functions, evidence theory, distance, combination rules, information source meta-data.keywords

\section{Introduction}

The theory of belief functions [9, 26] is a framework for reasoning under uncertainty and imprecision. It encompasses probability theory, possibility theory and Cantor's set theory. Approaches relying on the theory of belief functions are often called evidential because collected data are seen as pieces of evidence allowing the definition of some set functions describing the state of knowledge

*M. Loudahi, J. Klein, J-M. Vannobel and O. Colot are with Lille1 University and CRIStAL UMR CNRS 9189, Lille, France (e-mail: firstname.lastname@univ-lille1.fr).

${ }^{\dagger}$ Manuscript accepted for publication in IEEE Transactions on Cybernetics on january 2015. 
of a given information source concerning the problem at stake. The pieces of evidence collected by each source individually are called bodies of evidence.

In particular, in case of multiple information sources, it is sometimes necessary to determine if the contents of different bodies of evidence are similar or not. A distance between bodies of evidence can achieve this goal and such distances are called evidential distances.

Defining a meaningful evidential distance is a challenging task as dissimilarity has its roots in two main aspects: uncertainty and imprecision. For instance, the statement John is 30 years old is different in terms of uncertainty from the statement John is 30 years old with probability 0.6. The statement John is 30 years old is different in terms of imprecision from the statement John is in his thirties. It is obvious that a positive distance value is justified in both of these comparisons but it is far less obvious to determine if one of them should prevail or not. When introducing evidential distances, authors do not always justify their approach with respect to uncertainty or imprecision although their influences are latent. To some extent, defining evidential distances is thus an over-constrained problem with many possible heterogeneous solutions depending on a targeted purpose. Such purposes can be mathematical properties or applications. This accounts for the vast literature dealing with evidential distances.

The most popular evidential distance is Jousselme distance [16. Belief functions are set functions and their domain has a poset structure. Jousselme distance is a full metric and takes explicitly into account this poset structure. Prior to Jousselme et al. approach, people used to resort to pseudo-metrics [30, 22, 34, 32, 12 and [1. One full metric [15, 19] was introduced before Jousselme distance and used inside an optimization algorithm. Discussing its relevance for assessing belief function closeness was not in the scope of that paper. More recently, Diaz et al. introduced in 10] several metrics in the same vein as Jousselme distance but with different appraisals of structural aspects.

Most of the time, evidential distances are application-driven. For instance, Zouhal and Denœux [34] use a nearest neighbor classifier where neighbor testimonies are seen as bodies of evidence. The parameters involved in their evidential approach are optimized by minimizing a dissimilarity between these bodies. Fixen and Mahler [12 introduce a pseudo-metric to evaluate the performances of their classifier whose outputs are bodies of evidence. In [11 and 31, sensor data are represented as bodies of evidence and the reliability of the sensor is assessed using distances. A common issue when working in the framework of belief functions consists in approximating a body of evidence in order to obtain another one with desired properties or in order to reduce computation load. A convenient way to perform such approximations is to resort to distance minimization as proposed in [15, 1, 33, 32, 5, 17, or [8.

past decade.

Jousselme and Maupin [17] analyzed and surveyed the evidential distance literature and clarified the advantages and limitations of evidential distances. Based on their comments, we formalized in 21] a number of desirable mathematical properties for evidential distances and proposed specific distances possessing 
these properties. These distances are obtained using the norm of the difference between matrices encoding bodies of evidence. In particular, one of these new distances has similar properties as Jousselme distance while exhibiting a consistent behavior with respect to the conjunctive rule of combination. In this article, we continue our effort toward the definition of meaningful evidential distances by investigating the properties of distances derived using other matrices than those considered in 21].

After providing the necessary definitions and background related to the theory of belief functions in section 2, extended families of matrix-based evidential distances are presented in section 3 . These families are infinite and are defined using evidential matrices that are linked with combination rules known as $\alpha$ junctions. In section 4 the consistency of the proposed distances with respect to $\alpha$-junctions is investigated. Let $\mathcal{B}_{0}, \mathcal{B}_{1}$ and $\mathcal{B}_{2}$ denote three bodies of evidence. In this paper, we say that an evidential distance is consistent with a combination rule if the distance between $\mathcal{B}_{1}$ and $\mathcal{B}_{2}$ is greater or equal to the distance between $\left\{\mathcal{B}_{1}\right.$ combined with $\left.\mathcal{B}_{0}\right\}$ and $\left\{\mathcal{B}_{2}\right.$ combined with $\left.\mathcal{B}_{0}\right\}$. In section 5, we discuss the ability of the proposed distances to take the poset structure of belief functions domain into account. The importance of this aspect is stressed in [17. Finally, in section [6] evidential distances are also studied under a new perspective: the influence of meta-information. Meta-information is made of contextual data on the information sources. Various scenarios on the truthfulness of sources are considered.

\section{Belief function framework: notations and def- initions}

In this section, some mathematical notations for classical belief function concepts are given. The reader is expected to be familiar with belief function basics and consequently some definitions are not detailed. More material on belief functions basics is found for instance in [3. Special care is given to a reminder on $\alpha$-junctions and matrix calculus as part of the theory of belief functions.

\subsection{Belief function basics}

Suppose $\Omega=\left\{\omega_{i}\right\}_{i=1}^{n}$ is the finite domain of a given parameter $\theta$, i.e. the set of possible and unknown values $\omega_{i}$ of $\theta$. Suppose also $S_{i}$ is a source of information that collected a body of evidence $\mathcal{B}_{i}$ regarding the actual value of $\theta$. The set function representing all pieces of evidence in $\mathcal{B}_{i}$ is called a mass function and is denoted by $m_{i}$. The power set $2^{\Omega}$ is the set of all subsets of $\Omega$ and it is the domain of mass functions. For any $A \in 2^{\Omega}$, the cardinality of this set is denoted by $|A|$ and we thus have $|\Omega|=n$. The cardinality of $2^{\Omega}$ is denoted by $N=2^{n}$. Mass functions have $[0,1]$ as co-domain and they sum to one: $\sum_{A \in 2^{\Omega}} m_{i}(A)=1$. A focal element of a mass function $m_{i}$ is a set $A \subseteq \Omega$ such that $m_{i}(A)>0$, meaning that $\mathcal{B}_{i}$ contains evidence supporting the event 
$\{\theta \in A\}$. A mass function having only one focal element $A$ is called a categorical mass function and is denoted by $m_{A}$. The categorical mass function $m_{\Omega}$ is called the vacuous mass function because it carries no information. As explained in [6], a simple mass function is the convex combination of $m_{\Omega}$ with a categorical mass function $m_{A}$ with $A \neq \Omega$.

In the theory of belief functions, several alternative set functions are commonly used for evidence representation. In this paper, only the plausibility function $p l_{i}$ will be used. This set function is the conjugate of the inverse Möbius of the mass function $m_{i}$. The plausibility function $p l_{i}$ is in one-to-one correspondence with the mass function $m_{i}$ as it can be seen from the following formula :

$$
p l_{i}(A)=\sum_{\substack{X \subseteq \Omega \\ X \cap A \neq \emptyset}} m_{i}(X), \forall A \in 2^{\Omega} .
$$

Another useful concept is the negation (or complement) $\bar{m}_{i}$ of a mass function $m_{i}$. The function $\bar{m}_{i}$ is such that $\forall A \subseteq \Omega, \bar{m}_{i}(A)=m_{i}(\bar{A})$ with $\bar{A}=\Omega \backslash A$.

Besides, a mass function can be viewed as special kind of interval-valued probability distribution 9. As part of the Transferable Belief Model, Smets 29] introduced the pignistic transform which is one way to retrieve a probability distribution with values inside the intervals specified by a mass function. This distribution is called the pignistic probability distribution betp $p_{i}$ and it is obtained from $m_{i}$ as follows : $\forall \omega \in \Omega$,

$$
\operatorname{betp}_{i}(\omega)=\sum_{A \subseteq \Omega, \omega \in A} \frac{1}{|A|} \frac{m_{i}(A)}{1-m_{i}(\emptyset)}
$$

\subsection{Mass function combination using $\alpha$-junctions}

In this subsection, a brief presentation of $\alpha$-junctions 27 is proposed. Suppose two sources of information $S_{1}$ and $S_{2}$ have gathered pieces of evidence allowing them to define two mass functions $m_{1}$ and $m_{2}$ respectively. Evidential combination rules address the problem of aggregating these two functions into a single one synthesizing both of the initial evidence bodies. Let $f$ be a combination operator for mass functions, i.e. $m_{12}=f\left(m_{1}, m_{2}\right)$ with $m_{12}$ a mass function depending only on $m_{1}$ and $m_{2}$. Such an operator is an $\boldsymbol{\alpha}$-junction if it possesses the following properties:

- Linearity $1: \forall \lambda \in[0,1], f\left(m, \lambda m_{1}+(1-\lambda) m_{2}\right)=\lambda f\left(m, m_{1}\right)+(1-\lambda) f\left(m, m_{2}\right)$,

- Commutativity: $f\left(m_{1}, m_{2}\right)=f\left(m_{2}, m_{1}\right)$,

- Associativity: for any additional mass function $m_{3}, f\left(f\left(m_{1}, m_{2}\right), m_{3}\right)=$ $f\left(m_{1}, f\left(m_{2}, m_{3}\right)\right)$,

- Neutral element: $\exists m_{e} \mid \forall m, f\left(m, m_{e}\right)=m$,

\footnotetext{
${ }^{1}$ The operator is linear on the vector space spanned by categorical mass functions but the output of the operator remains a mass function only in case of convex combination.
} 
- Anonymity: for any mapping $\sigma: 2^{\Omega} \longrightarrow 2^{\Omega}$ such that its restriction on $\Omega$ is a permutation and $\sigma(X)=\bigcup_{x \in X} \sigma(x)$ when $|X|>1$, we have $f\left(m_{1} \circ \sigma, m_{2} \circ \sigma\right)=m_{12} \circ \sigma$,

- Context preservation: $p l_{1}(X)=0$ and $p l_{2}(X)=0 \Longrightarrow p l_{12}(X)=0$.

In short, $\alpha$-junctions are thus linear combination rules that do not depend on the order in which pieces of evidence are processed. The justification behind these properties are detailed in 27. In the same article, Smets proves that the neutral element $m_{e}$ can only be either $m_{\emptyset}$ or $m_{\Omega}$. Depending on this, two subfamilies arise: the $\alpha$-disjunctive rules denoted by (1) ${ }^{\alpha}$ and the $\alpha$-conjunctive rules denoted by $\left(^{\alpha}{ }^{\alpha}\right.$. For both of these families, Pichon and Denœux [24] provided the following computation formulae: $\forall X \subseteq \Omega, \forall \alpha \in[0,1]$

$$
\begin{aligned}
m_{1 \cap \alpha_{2}}(X) & =\sum_{(A \cap B) \cup(\bar{A} \cap \bar{B} \cap C)=X} m_{1}(A) m_{2}(B) \alpha^{|\bar{C}|} \bar{\alpha}^{|C|}, \\
m_{1 \cup^{\alpha} 2}(X) & =\sum_{(A \triangle B) \cup(A \cap B \cap C)=X} m_{1}(A) m_{2}(B) \alpha^{|C|} \bar{\alpha}^{|\bar{C}|},
\end{aligned}
$$

with $\bar{\alpha}=1-\alpha$ and $\Delta$ the set symmetric difference. It is noteworthy that, if $\alpha=1$, the classical conjunctive and disjunctive rules are retrieved. We denote

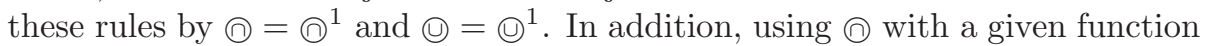
$m_{1}$ and a given categorical function $m_{B}$ is tantamount to conditioning $m_{1}$ on $B$. The following notations will be used when combining $m_{B}$ with a function $m_{1}$ :

- $m_{1 \mid B}:=m_{1} @ m_{B}$, this mass function is sometimes referred to as $m_{1}$ given $B$,

- $m_{1 \cap^{\alpha} B}:=m_{1} \bigcirc^{\alpha} m_{B}$,

- $m_{1 \cup^{\alpha} B}:=m_{1} \Theta^{\alpha} m_{B}$,

- $m_{\left.1\right|^{\alpha} B}$ stands for the combination of $m_{1}$ and $m_{B}$ using an $\alpha$-junction when distinguishing conjunctive and disjunctive cases is unnecessary.

\subsection{Meta-information and $\alpha$-junctions}

The interpretation of $\alpha$-junctions is related to information items concerning the truthfulness of the sources $S_{1}$ and $S_{2}$. In an information fusion context, such items are known as meta-information and truthfulness is a special kind of meta-information. Actually, several forms of truthfulnesses can be observed in practice but regarding $\alpha$-junctions the following definition is retained: $S_{i}$ is untruthful if it supports the opposite of what it knows to be true.

Depending on the truthfulness of sources, very different decisions can be made in the end, which accounts for the importance of taking meta-information into account in information fusion problems. In general, our knowledge about the 
truthfulness of each source is imprecise and uncertain and it is therefore expressed as a mass function on a meta-domain $\mathcal{T}_{i}$. In [23, Pichon explains that an element $t_{C}^{i} \in \mathcal{T}_{i}$ is understood as the fact that $S_{i}$ is truthful when it supports $\{\theta \in C\}$ and it is untruthful when it supports $\{\theta \in \bar{C}\}$. Let us provide a simple example when everything is deterministic:

Example 1. Suppose that $|\Omega|=4$ and the meta-data concerning $S_{i}$ is that $t_{C}^{i}$ has probability 1 with $C=\left\{\omega_{1}, \omega_{2}\right\}$. If the source of information $S_{i}$ delivers only one certain piece of evidence $\theta \in A=\left\{\omega_{2}, \omega_{3}\right\}$, then four different situations are encountered:

- The source gives support to $\omega_{2}$ and can be trusted about $\omega_{2}$. We conclude that $\omega_{2}$ is a possible value for $\theta$.

- The source gives no support to $\omega_{1}$ and can be trusted about $\omega_{1}$. We conclude that $\omega_{1}$ is a not possible value for $\theta$.

- The source gives support to $\omega_{3}$ but cannot be trusted about $\omega_{3}$. We conclude that $\omega_{3}$ is not a possible value for $\theta$.

- The source gives no support to $\omega_{4}$ but cannot be trusted about $\omega_{4}$. We conclude that $\omega_{4}$ is a possible value for $\theta$.

All in all, the testimony of the source is $\theta \in A$ but given the meta-data, the actual testimony is $\theta \in \overline{A \Delta C}$.

When considering a pair of sources $\left(S_{1} ; S_{2}\right)$, meta-events belong to $\mathcal{T}_{1} \times \mathcal{T}_{2}$. Pichon also proves that:

- for $\alpha$-conjunctions, the underlying meta-information is that each metaevent $\{$ either both sources are fully truthful or they both lie about $\bar{C}\}=$ $\left\{\left(t_{\Omega}^{1} ; t_{\Omega}^{2}\right) ;\left(t_{C}^{1} ; t_{C}^{2}\right)\right\}$ has probability $\alpha^{|C|} \bar{\alpha}^{|\bar{C}|}$.

- for $\alpha$-disjunctions, the underlying meta-information is that each metaevent \{one source is fully truthful while the other one lies at least about $C\}=\bigcup_{X \subseteq \bar{C}}\left\{\left(t_{\Omega}^{1} ; t_{X}^{2}\right) ;\left(t_{X}^{1} ; t_{\Omega}^{2}\right)\right\}$ has probability $\alpha^{|\bar{C}|} \bar{\alpha}^{|C|}$.

In particular, when $\alpha=1$, the above probabilities are null whenever $C \neq \Omega$ in the conjunctive case and whenever $C \neq \emptyset$ in the disjunctive case. The metainformation thus reduces to:

- for the conjunctive rule, the event $\{$ both sources are fully truthful $\}$ has probability 1 .

- for the disjunctive rule, the event \{at least one of the sources is fully truthful\} has probability 1 .

Note that $\alpha$-junctions are a particular case of a combination process introduced in 25] where a general framework for reasoning under various meta-information is formalized. 


\subsection{Belief functions and matrix calculus}

Mass functions can be viewed as vectors belonging to the vector space $\mathbb{R}^{N}$ with categorical mass functions as base vectors. Since mass functions sum to one, the set of mass functions is the simplex $\mathcal{S}$ in that vector space whose vertices are the base vectors $\left\{m_{A}\right\}_{A \subset \Omega}$. This simplex is also called mass space [6]. In this paper, the following notations and conventions are used :

- Vectors are column vectors and are written in bold small letters. The operator vec maps a set function or a distribution to its vector form. For instance, $\mathbf{m}_{i}=\mathbf{v e c}\left(m_{i}\right)$ is the mass vector corresponding to a mass function $m_{i}$. The length of mass vectors is $N$. The $j_{A}{ }^{\text {th }}$ element of a mass vector $\mathbf{m}_{i}$ is such that $\mathbf{m}_{i}\left(j_{A}\right)=m_{i}(A)$ with $j_{A}$ the integer index of the set $A$ according to the binary order. The binary order 28 is a common way to index elements of $2^{\Omega}$ without supposing any order on $\Omega$.

- Matrices are written in bold capital letters. They are square and their size is $N \times N$. A matrix can be represented by $\mathbf{X}=\left[X\left(i_{A}, j_{B}\right)\right]$, or alternatively by the notation $\mathbf{X}=[X(A, B)], \quad \forall A, B \in \Omega$. The row and column indexes $i_{A}$ and $j_{B}$ are those corresponding to the subsets $A$ and $B$ using the binary order.

- $\mathbf{I}$ is the identity matrix.

- $\mathbf{1}$ is the all-ones matrix.

- $\mathbf{J}$ is the matrix with null components except those on the anti-diagonal which are equal to 1 . $\mathbf{J}$ is a permutation matrix reversing lines in case of right-handed product and reversing columns in case of left-handed product.

Matrix calculus as part of the theory of belief functions is especially interesting when it comes to mass function $\alpha$-junctive combination. In [28] and 27, Smets shows that equation (3) and (4) can be written as a product between a matrix and a mass function vector. Let $\mathbf{K}_{1, \alpha}^{\cap}$ be a matrix such that $K_{1, \alpha}^{\cap}(A, B)=$ $m_{1 \cap^{\alpha} B}(A)$ and $\mathbf{K}_{1, \alpha}^{\cup}$ a matrix such that $K_{1, \alpha}^{\cup}(A, B)=m_{1 \cup^{\alpha} B}(A)$. One has:

$$
\begin{aligned}
& \mathbf{m}_{1 \cap^{\alpha} 2}=\mathbf{K}_{1, \alpha}^{\bigcap} \mathbf{m}_{2}, \\
& \mathbf{m}_{1 \cup^{\alpha} 2}=\mathbf{K}_{1, \alpha}^{\cup} \mathbf{m}_{2} .
\end{aligned}
$$

These matrices are also in one-to-one correspondence with the mass function $m_{1}$. We call $\mathbf{K}_{1, \alpha}^{\cap}$ and $\mathbf{K}_{1, \alpha}^{\cup}$ the $\boldsymbol{\alpha}$-specialization and $\boldsymbol{\alpha}$-generalization matrices corresponding to $m_{1}$. In general, all such matrices will be called evidential matrices. When it is not necessary to stress the dependency of evidential matrices on $\alpha$ and on the conjunctive/disjunctive cases, an evidential matrix is denoted by $\mathbf{K}_{1}$ for the sake of equation concision.

Each element of $\mathbf{K}_{1}$ represents the mass assigned to a set $A$ after learning that $\{\theta \in B\}: K_{1}(A, B)=m_{\left.1\right|^{\alpha} B}(A)$. In other words, $\mathbf{K}_{1}$ does not only represent the current state of belief depicted by $m_{1}$ but also all reachable states from 
$m_{1}$ through an $\alpha$-junctive conditioning. From a geometric point of view [4, each column of an evidential matrix $\mathbf{K}_{1}$ corresponds to the vertex of a polytope $\mathcal{P}_{1}$, called the conditional subspace of $m_{1}$. Example 2 illustrates this latter remark.

Example 2. Let $|\Omega|=2$ and $m_{1}=\bar{\lambda} m_{\left\{\omega_{1}\right\}}+\lambda m_{\Omega}$ with $\lambda \in[0 ; 1]$ and $\bar{\lambda}=1-\lambda$. In the conjunctive case, we have :

$$
\mathbf{K}_{1, \alpha}^{\cap}=\left(\begin{array}{cccc}
\bar{\lambda} \alpha+\lambda & 0 & \bar{\lambda} & 0 \\
0 & \bar{\lambda} \alpha+\lambda & 0 & \bar{\lambda} \\
\bar{\lambda} \bar{\alpha} & 0 & \lambda & 0 \\
0 & \bar{\lambda} \bar{\alpha} & 0 & \lambda
\end{array}\right) .
$$

The four column vectors of $\mathbf{K}_{1, \alpha}^{\bigcap}$ are (from left to right) : $\mathbf{m}_{1 \cap^{\alpha} \emptyset}, \mathbf{m}_{1 \cap^{\alpha}\left\{\omega_{1}\right\}}$, $\mathbf{m}_{1 \cap \alpha\left\{\omega_{2}\right\}}$, and $\mathbf{m}_{1}$. By definition the polytope $\mathcal{P}_{1}$ is the following subset of $\mathcal{S}$ :

$$
\left\{m \in \mathcal{S} \mid m=\sum_{A \subseteq \Omega} \lambda_{A} \mathbf{m}_{1 \cap^{\alpha} A}, \sum_{A \subseteq \Omega} \lambda_{A}=1, \lambda_{A} \in[0 ; 1]\right\} .
$$

Any mass function $m \in \mathcal{P}_{1}$ is the result of the combination of $m_{1}$ with another mass function using a given $\alpha$-junction. Evidential matrices are consequently relevant candidates for assessing dissimilarities between bodies of evidence in compliance with $\alpha$-junctions.

Most importantly, if $\mathbf{K}_{1}$ and $\mathbf{K}_{2}$ are two evidential matrices and if $\mathbf{K}_{12}$ denotes the matrix corresponding to the $\alpha$-junction of $m_{1}$ with $m_{2}$, then one has:

$$
\mathbf{K}_{12}=\mathbf{K}_{1} \mathbf{K}_{2} .
$$

Moreover, the transpose of any evidential matrix $\mathbf{K}$ is a stochastic matrix, meaning that all lines sum to one: ${ }^{\mathbf{t}} \mathbf{K} \mathbf{1}=\mathbf{1}$, with ${ }^{\mathbf{t}} \mathbf{K}$ the transpose matrix of $\mathbf{K}$. Finally, $\mathbf{K}_{A}$ will denote the evidential matrix corresponding to the categorical mass function $m_{A}$.

\section{Distances in evidence theory}

In this section, we will first recall the definitions of some existing distances in the theory of belief functions. We will focus on various kinds of evidential dissimilarities without aiming at providing an exhaustive review. Afterward, new matrix-based evidential distances will be introduced in connection with $\alpha$-junctions.

\subsection{Vector-based evidential distances}

A distance, or metric, provides a positive real value assessing the discrepancies between two elements. Let us first give a general definition of such an application when the compared vectors are mass functions: 
Definition 1. Given a domain $\Omega$ and its related mass space $\mathcal{S}$, a mapping $d: \mathcal{S} \times \mathcal{S} \longrightarrow[0, a]$ with $a \in \mathbb{R}^{+}$is a distance between two mass functions $m_{1}$ and $m_{2}$ defined on $\Omega$ if the following properties hold:

- Symmetry : $d\left(m_{1}, m_{2}\right)=d\left(m_{2}, m_{1}\right)$,

- Definiteness : $d\left(m_{1}, m_{2}\right)=0 \Leftrightarrow m_{1}=m_{2}$,

- Triangle inequality : $d\left(m_{1}, m_{2}\right) \leq d\left(m_{1}, m_{3}\right)+d\left(m_{3}, m_{2}\right)$.

If the mapping fails to possess some of the above properties, then it degrades into unnormalized distance, dissimilarity or pseudo-distance. Only full metrics are able to provide a positive finite value that matches the intuitive notion of $g a{ }^{2}$ between elements of a given space.

If $a \neq+\infty$, then the distance is bounded and if in addition $a=1$, the distance is normalized. Provided that an evidential distance $d$ is bounded, this distance can be normalized by dividing it with $\rho=\max _{m_{1}, m_{2} \in \mathcal{S}} d\left(m_{1}, m_{2}\right)$. By definition, $\rho$ is the diameter of $\mathcal{S}$. A result in convex geometry states that the diameter of a simplex is actually the length of its largest edge. Consequently, the following lemma holds:

Lemma 1. Let $d$ be a bounded evidential distance. Let $\tilde{d}$ be the evidential distance defined by $\tilde{d}\left(m_{1}, m_{2}\right)=\frac{d\left(m_{1}, m_{2}\right)}{\rho}$ with $\rho=\max _{A, B \in 2^{\Omega}} d\left(m_{A}, m_{B}\right)$. Then, $\tilde{d}$ is a normalized evidential distance.

In evidence theory, two main families of mass function dissimilarities have been introduced, namely: direct and indirect dissimilarities. Direct measures are those defined on the product space $\mathcal{S} \times \mathcal{S}$ and thus complying with the geometrical interpretation of belief functions [6]. Concerning direct dissimilarities, the most widely used is Jousselme distance [16]. It is based on an inner product relying on a similarity matrix. This distance is given by:

$$
d_{J}\left(m_{1}, m_{2}\right)=\sqrt{\frac{1}{2}{ }^{t}\left(\mathbf{m}_{\mathbf{1}}-\mathbf{m}_{\mathbf{2}}\right) \mathbf{D}\left(\mathbf{m}_{\mathbf{1}}-\mathbf{m}_{\mathbf{2}}\right)},
$$

with $\mathbf{D}$ the Jaccard similarity matrix between focal elements. Its components are:

$$
D(A, B)= \begin{cases}1 & \text { if } A=B=\emptyset \\ \frac{|A \cap B|}{|A \cup B|} & \text { otherwise }\end{cases}
$$

The success of Jousselme distance is explained by the fact that, thanks to the matrix $\mathbf{D}$, the dependencies between the base vectors of $\mathcal{S}$ are taken in account. Consequently, the poset structure of $2^{\Omega}$ has an impact on distance values, allowing a better match with the user's expectations.

Many other evidential distances are defined similarly by substituting matrix $\mathbf{D}$

\footnotetext{
${ }^{2}$ This term was used by Frechet [13] in his early works on metric spaces, i.e. spaces endowed with a distance.
} 
with another matrix evaluating the similarity between base vectors in different ways [10, 6]. Experimental material in [16] shows that these distances are highly correlated to $d_{J}$.

As for indirect dissimilarities, each mass function is first transformed into a vector belonging to a new space of representation $\mathcal{X} \subset \mathbb{R}^{M}$ related to another uncertainty theory. Afterwards, a distance measure is computed in this new representation space. When these transforms are lossy $(M<N)$, indirect dissimilarities are not definite, hence none of them is a full metric. The most frequently used transform for indirect dissimilarities is the pignistic transform as proposed by Tessem 32 or Liu [20]. Tessem dissimilarity is defined as follows:

$$
d_{T}\left(m_{1}, m_{2}\right)=\left\|\mathbf{b e t}_{1}-\mathbf{b e t}_{2}\right\|_{\infty}
$$

with $\operatorname{bet}_{i}=\operatorname{vec}\left(\right.$ betp $\left._{i}\right)$ the vector corresponding to the pignistic probability distribution obtained from $m_{i}$.

In the same vein, Zouhal and Denœux 34 also define a dissimilarity measure based on the pignistic transform but they use the euclidean $L^{2}$ norm instead of the $L^{\infty}$ norm:

$$
d_{Z D}\left(m_{1}, m_{2}\right)=\| \text { betp }_{1}-\text { betp }_{2} \|_{2} .
$$

Other indirect dissimilarities [14] can be obtained in a space spanned by fuzzy set distributions. The vector-based distances cited in this subsection constitute a small sample of those found in the literature. However, this is a representative sample with respect to the diversity of evidential dissimilarities. They are therefore relevant candidates for a comparison with the new distances that will be introduced in the next subsection.

\subsection{Matrix-based evidential distances}

Since evidential matrices and mass functions are in one-to-one correspondence, assessing the distance between bodies of evidence can also be performed using distances between evidential matrices. The set of $N \times N$ matrices is denoted by $\mathcal{M}_{N}$ and has the algebraic properties of a vector space as well. Consequently, matrix distances are not much different from vector distances. In this paper, we focus on matrix distances induced by matrix norms. A matrix norm is defined as follows:

Definition 2. A matrix norm $\|\cdot\|$ is a mapping defined on $\mathcal{M}_{n} \longrightarrow \mathbb{R}^{+}$ satisfying the following conditions: $\forall, \mathbf{A}$ and $\mathbf{B} \in \mathcal{M}_{N}$

1. $\|\mathbf{A}\|=\mathbf{0} \Leftrightarrow \mathbf{A}=\mathbf{0}$,

2. $\|\lambda \mathbf{A}\|=|\lambda| \cdot\|\mathbf{A}\|$, for all $\lambda \in \mathbb{R}^{+}$,

3. $\|\mathbf{A}+\mathbf{B}\| \leq\|\mathbf{A}\|+\|\mathbf{B}\|$,

A matrix norm is sub-multiplicative, if in addition one has:

$$
\|\mathbf{A} \mathbf{B}\| \leq\|\mathbf{A}\|\|\mathbf{B}\|
$$


Any norm induces a distance defined as the norm of the difference between a pair of elements. Alleging that a matrix norm is bounded for evidential matrices, we thus introduce the following families of normalized evidential distances:

Definition 3. An $\alpha$-specialization distance $d$ is a mapping such that there exists a bounded matrix norm $\|$.$\| and an \alpha$-conjunction $\cap^{\alpha}$ with:

$$
\begin{aligned}
& d: \mathcal{S} \times \mathcal{S} \rightarrow[0,1] \\
& m_{1} \times m_{2} \rightarrow \frac{1}{\rho}\left\|\mathbf{K}_{1, \alpha}^{\cap}-\mathbf{K}_{2, \alpha}^{\cap}\right\|
\end{aligned}
$$

$\mathbf{K}_{i, \alpha}^{\cap}$ is the $\alpha$-specialization matrix corresponding to $m_{i}$ and $\rho=\max _{A, B \in 2^{\Omega}}\left\|\mathbf{K}_{A, \alpha}^{\cap}-\mathbf{K}_{B, \alpha}^{\cap}\right\|$ is a normalization factor.

Definition 4. An $\boldsymbol{\alpha}$-generalization distance $d$ is a mapping such that there exists a bounded matrix norm $\|$.$\| and an \alpha$-disjunction (1) ${ }^{\alpha}$ with:

$$
\begin{aligned}
d: \mathcal{S} \times \mathcal{S} & \rightarrow[0,1] \\
m_{1} \times m_{2} & \rightarrow \frac{1}{\rho}\left\|\mathbf{K}_{1, \alpha}^{\cup}-\mathbf{K}_{2, \alpha}^{\cup}\right\| .
\end{aligned}
$$

$\mathbf{K}_{i, \alpha}^{\cup}$ is the $\alpha$-generalization matrix corresponding to $m_{i}$ and $\rho=\max _{A, B \in 2^{\Omega}}\left\|\mathbf{K}_{A, \alpha}^{\cup}-\mathbf{K}_{B, \alpha}^{\cup}\right\|$ is a normalization factor.

The family of $\alpha$-specialization distances is an extension of the family introduced in 21] which corresponds to the $\alpha=1$ case. Among existing matrix norms, we focus in this article on the most frequently used ones, namely: $L^{k}$ norms and operator norms.

$L^{k}$ matrix norms are also known as entry-wise norms. Since matrices are elements of the vector space $\mathcal{M}_{N}$, the definition of $L^{k}$ matrix norms is the following:

$$
\|\mathbf{A}\|_{k}=\left(\sum_{1 \leq j \leq n} \sum_{1 \leq i \leq n}\left|\mathbf{A}_{i j}\right|^{k}\right)^{\frac{1}{k}} .
$$

Both $L^{k}$ vector norms and $L^{k}$ matrix norms are denoted by $\|\cdot\|_{k}$. They are easily distinguished since vectors are in small letters and matrices are in capital letters.

The $k$-operator norm $\|\cdot\|_{o p k}$, also known as induced norm, is defined for any matrix $\mathbf{A} \in \mathcal{M}_{N}$ as follows:

$$
\|\mathbf{A}\|_{o p k}=\max _{\mathbf{x} \in \mathbb{R}^{N}, \mathbf{x} \neq \mathbf{0}} \frac{\|\mathbf{A} \mathbf{x}\|_{k}}{\|\mathbf{x}\|_{k}}=\max _{\mathbf{x} \in \mathbb{R}^{N},\|\mathbf{x}\|_{k}=1}\|\mathbf{A} \mathbf{x}\|_{k} .
$$

with $\|\cdot\|_{k}$ the classical $L^{k}$ vector norm. In particular, the 1-operator norm writes as:

$$
\|\mathbf{A}\|_{o p 1}=\max _{1 \leq j \leq n} \sum_{1 \leq i \leq n}\left|\mathbf{A}_{i j}\right|
$$


It can be noted that any evidential matrix $\mathbf{K}$ is such that $\|\mathbf{K}\|_{o p 1}=1$. In the sequel, $d_{k}$ denotes the $\alpha$-specialization or $\alpha$-generalization distance relying on the $L^{k}$ matrix norm. The notation $d_{o p k}$ is used when the distance relies on the $k$-operator norm. In the same fashion as for evidential matrices, the value of $\alpha$ and the conjunctive or disjunctive nature of these distances are given in exponent when necessary.

A first result concerning the newly introduced distances is that there is a duality between $\alpha$-specialization distances and $\alpha$-generalization distances that has its roots in the De Morgan relations proved in 28. The following proposition formalizes this duality.

Proposition 1. Suppose $\alpha \in[0,1]$. Let $d^{\cap}$ be an $\alpha$-specialization distance with respect to the $\alpha$-conjunctive rule $\AA^{\alpha}$ and relying on an operator or $L^{k}$ matrix norm. Let $d^{\cup}$ be an $\alpha$-generalization distance with respect to the $\alpha$-disjunctive rule (1) ${ }^{\alpha}$ and relying on the same norm. For any mass functions $m_{1}$ and $m_{2}$ on a domain $\Omega$, one has:

$$
d^{\cap}\left(m_{1}, m_{2}\right)=d^{\cup}\left(\bar{m}_{1}, \bar{m}_{2}\right) .
$$

Proof. Let $\mathbf{K}_{i}^{\bigcap}$ and $\mathbf{K}_{i}^{\cup}$ be the $\alpha$-specialization matrix and the $\alpha$-generalization matrix of $m_{i}$ with $i \in\{1 ; 2\}$. Let $\overline{\mathbf{K}}_{i}^{\cap}$ and $\overline{\mathbf{K}}_{i}^{\cup}$ be the $\alpha$-specialization matrix and the $\alpha$-generalization matrix of $\bar{m}_{i}$ with $i \in\{1 ; 2\}$. According to theorem 12.1 in [28], we have $\mathbf{K}_{i}^{\bigcap}=\mathbf{J} \overline{\mathbf{K}}_{i}^{\cup} \mathbf{J}$ with $\mathbf{J}$ the binary anti-diagonal matrix. It follows that:

$$
\begin{aligned}
d^{\cap}\left(m_{1}, m_{2}\right) & =\frac{1}{\rho}\left\|\mathbf{K}_{1}^{\cap}-\mathbf{K}_{2}^{\cap}\right\|, \\
& =\frac{1}{\rho}\left\|\mathbf{J} \overline{\mathbf{K}}_{1}^{\cup} \mathbf{J}-\mathbf{J} \overline{\mathbf{K}}_{2}^{\cup} \mathbf{J}\right\|, \\
& =\frac{1}{\rho}\left\|\mathbf{J}\left(\overline{\mathbf{K}}_{1}^{\cup}-\overline{\mathbf{K}}_{2}^{\cup}\right) \mathbf{J}\right\| .
\end{aligned}
$$

Since $\mathbf{J}$ is a permutation matrix and that any $L^{k}$ matrix norm or operator norm computation is invariant to column or line relabeling, one has $\left\|\mathbf{J}\left(\overline{\mathbf{K}}_{1}^{\cup}-\overline{\mathbf{K}}_{2}^{\cup}\right) \mathbf{J}\right\|=$ $\left\|\overline{\mathbf{K}}_{1}^{\cup}-\overline{\mathbf{K}}_{2}^{\cup}\right\|=\rho d^{\cup}\left(\bar{m}_{1}, \bar{m}_{2}\right)$.

Proposition 1 sheds light on ties between $\alpha$-specialization distances and $\alpha$ generalization distances. When $\alpha \in\{0 ; 1\}$, it appears that these ties are stronger as illustrated by Lemma 2 .

Lemma 2. Let $d^{\cap, \alpha}$ be an $\alpha$-specialization distance with respect to the $\alpha$ conjunctive rule $\left(^{\alpha}\right.$ and relying on an operator or $L^{k}$ matrix norm. Let $d^{\cup, \alpha}$ be an $\alpha$-generalization distance with respect to the $\alpha$-disjunctive rule $\left(\mathbb{C}^{\alpha}\right.$ and relying on the same norm. For any mass functions $m_{1}$ and $m_{2}$ on a domain $\Omega$, one has:

$$
\begin{aligned}
& d^{\cap, 0}=d^{\cup, 0}, \\
& d^{\cap, 1}=d^{\cup, 1} .
\end{aligned}
$$


The proof of Lemma 2 is given in appendix $\mathrm{A}$ This result shows that, for extreme values of $\alpha, \alpha$-specialization distances and $\alpha$-generalization distances are identical. Consequently, the underlying meta-information is treated in the same way in these special cases. The following example shows that this is not true when $\alpha \in] 0,1[$ :

Example 3. Suppose $\Omega$ is a domain and $X$ a subset of $\Omega$. Let $m$ be a mass function such that:

$$
m=0.3 m_{X}+0.5 m_{\bar{X}}+0.2 m_{\Omega} .
$$

Figure 1 shows $\alpha$-specialization and $\alpha$-generalization distances relying on the $L^{1}$ matrix norm between $m$ and $m_{X}$ when $|\Omega|=3$ and $|X|=2$.

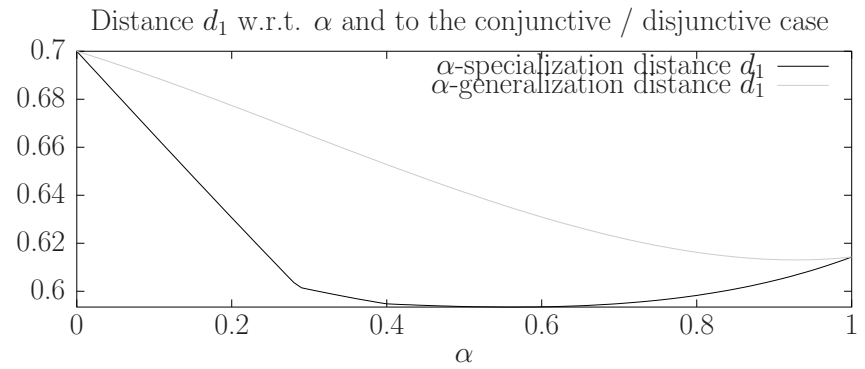

Figure 1: Different $\alpha$-specialization and $\alpha$-generalization distances relying on the $L^{1}$ matrix norm. These distances are computed between two given mass functions $m_{1}$ and $m_{2}$ such that $m_{1}=m_{X}$ and $m_{2}=0.3 m_{X}+0.5 m_{\bar{X}}+0.2 m_{\Omega}$, with $|\Omega|=3$ and $|X|=2$.

Besides, proposition 1 allows us to anticipate the fact that if an $\alpha$-specialization distance satisfies a given property then so does its $\alpha$-generalization counterpart. Concerning evidential distance properties, we argued in 21] that, on top of metric properties, two other properties are especially interesting. The definitions and justifications of each of them are given respectively in section 4 and 5 . We will also examine what $\alpha$-specialization distances and $\alpha$-generalization distances may satisfy these properties.

\section{Consistency with $\alpha$-junctions}

One way to formalize the consistency of an evidential distance with a combination rule is given by the following definition:

Definition 5. Let $\odot$ be a combination rule and $d$ an evidential distance. $d$ is said to be consistent with respect to $\odot$ if for any mass functions $m_{1}, m_{2}$ and $m_{3}$ on $\Omega$ :

$$
d\left(m_{1} \odot m_{3}, m_{2} \odot m_{3}\right) \leq d\left(m_{1}, m_{2}\right) .
$$


Suppose each mass function $m$ can be decomposed into elementary pieces of evidence such that their aggregation using a combination rule gives $m$. For instance, Shafer [26] introduced the canonical decomposition under Dempster's rule of a particular class of mass functions into simple mass function sets. Using a consistent evidential distance, mass functions are all the closer as their decompositions involve identical elementary pieces of evidence.

\subsection{Results on the consistency of matrix-based evidential distances}

We provide several results concerning the consistency of $\alpha$-specialization distances and $\alpha$-generalization distances. The first one deals with these distances when the 1-operator norm is used:

Proposition 2. Any $\alpha$-specialization or $\alpha$-generalization distance $d_{o p 1}$ defined using the 1-operator norm is consistent with its corresponding $\alpha$-junctive combination rule.

Proof. Suppose $m_{1}, m_{2}$ and $m_{3}$ are three mass functions defined on $\Omega . \mathbf{K}_{1}, \mathbf{K}_{2}$ and $\mathbf{K}_{3}$ are their respective evidential matrices with respect to a given $\alpha$-junction denoted by $\odot^{\alpha}$. The 1-operator-norm has the sub-multiplicative property, i.e. for all matrices $\mathbf{A}$ and $\mathbf{B}$, one has:

$$
\|\mathbf{A B}\|_{o p 1} \leq\|\mathbf{A}\|_{o p 1} \cdot\|\mathbf{B}\|_{o p 1}
$$

One can thus write:

$$
\left\|\left(\mathbf{K}_{1}-\mathbf{K}_{2}\right) \mathbf{K}_{3}\right\|_{o p 1} \leq\left\|\mathbf{K}_{1}-\mathbf{K}_{2}\right\|_{o p 1} \cdot\left\|\mathbf{K}_{3}\right\|_{o p 1},
$$

Given that $\left\|\mathbf{K}_{3}\right\|_{o p 1}=1$, we have:

$$
\begin{aligned}
\left\|\left(\mathbf{K}_{1}-\mathbf{K}_{2}\right) \mathbf{K}_{3}\right\|_{o p 1} & \leq\left\|\mathbf{K}_{1}-\mathbf{K}_{2}\right\|_{o p 1}, \\
\Leftrightarrow d_{o p 1}\left(m_{1} \odot^{\alpha} m_{3}, m_{2} \odot^{\alpha} m_{3}\right) & \leq d_{o p 1}\left(m_{1}, m_{2}\right) .
\end{aligned}
$$

By definition, this latter inequality means that distance $d_{o p 1}$ is consistent with rule $\odot^{\alpha}$.

Another result holds when $\alpha$-specialization or $\alpha$-generalization distances are defined using the $L^{1}$ matrix norm. To prove this result, it is first necessary to introduce the following lemma:

Lemma 3. Suppose $m_{1}$ and $m_{2}$ are two mass functions defined on $\Omega$ and $A$ and $B$ are two subsets such that $A \subseteq B \subseteq \Omega$. Then, the following properties hold for any $\alpha$-conjunctive rule $\left(^{\alpha}\right.$ and any $\alpha$-disjunctive rule (1) ${ }^{\alpha}$

$$
\begin{aligned}
& \left\|\mathbf{m}_{1 \cap^{\alpha} A}-\mathbf{m}_{2 \cap^{\alpha} A}\right\|_{1} \leq\left\|\mathbf{m}_{1 \cap^{\alpha} B}-\mathbf{m}_{2 \cap^{\alpha} B}\right\|_{1}, \\
& \left\|\mathbf{m}_{1 \cup^{\alpha} A}-\mathbf{m}_{2 \cup^{\alpha} A}\right\|_{1} \geq\left\|\mathbf{m}_{1 \cup^{\alpha} B}-\mathbf{m}_{2 \cup^{\alpha} B}\right\|_{1} .
\end{aligned}
$$


The proof of Lemma 3 is given in appendix B. From this lemma, we deduce the following corollary:

Corollary 1. Suppose $m_{1}$ and $m_{2}$ are two mass functions defined on $\Omega$. Let $d_{o p 1}$ be an $\alpha$-specialization or $\alpha$-generalization distance defined using the 1-operator norm. We have:

$$
d_{o p 1}\left(m_{1}, m_{2}\right)=\frac{1}{\rho}\left\|\mathbf{m}_{1}-\mathbf{m}_{2}\right\|_{1} .
$$

This means that the 1-operator distance $d_{o p 1}$ is the $L^{1}$ distance between mass vectors for any $\alpha$. The proof of corollary 1 is given in appendix $[$, This corollary also implies that $\rho=2$ for $d_{o p 1}$. Equipped with these preliminary results, we are now able to provide the following proposition regarding the consistency of distances defined using the $L^{1}$ norm:

Proposition 3. Any $\alpha$-specialization or $\alpha$-generalization distance $d_{1}$ defined using the $L^{1}$ matrix norm is consistent with its corresponding $\alpha$-junctive combination rule.

Proof. Suppose $m_{1}, m_{2}$ and $m_{3}$ are three mass functions defined on $\Omega$. Suppose $\mathbf{K}_{1}, \mathbf{K}_{2}$ and $\mathbf{K}_{3}$ are their respective evidential matrices with respect to an $\alpha$ junction denoted by $\odot^{\alpha}$. The $L^{1}$ norm of a matrix is the sum over all columns of the $L^{1}$ norms of its column vectors:

$$
\left\|\left(\mathbf{K}_{1}-\mathbf{K}_{2}\right) \mathbf{K}_{3}\right\|_{1}=\sum_{A \subseteq \Omega}\left\|\mathbf{m}_{\left.13\right|^{\alpha} A}-\mathbf{m}_{\left.23\right|^{\alpha} A}\right\|_{1},
$$

with $m_{\left.i 3\right|^{\alpha} A}=m_{i} \odot^{\alpha} m_{A} \odot^{\alpha} m_{3}$. Besides, according to proposition 2] $d_{o p 1}$ is consistent with $\odot^{\alpha}$. Inequality (20) thus applies, and after multiplying both sides of this inequality by $\rho$, we have that for any $A \subseteq \Omega$ :

$$
\left\|\left(\mathbf{K}_{\left.1\right|^{\alpha} A}-\mathbf{K}_{\left.2\right|^{\alpha} A}\right) \mathbf{K}_{3}\right\|_{o p 1} \leq\left\|\mathbf{K}_{\left.1\right|^{\alpha} A}-\mathbf{K}_{\left.2\right|^{\alpha} A}\right\|_{o p 1},
$$

with $\mathbf{K}_{\left.i\right|^{\alpha} A}$ the evidential matrix corresponding to $m_{\left.i\right|^{\alpha} A}$ with respect to $\odot^{\alpha}$. Now applying corollary 1 on each side of the above inequality gives:

$$
\left\|\mathbf{m}_{\left.13\right|^{\alpha} A}-\mathbf{m}_{\left.23\right|^{\alpha} A}\right\|_{1} \leq\left\|\mathbf{m}_{\left.1\right|^{\alpha} A}-\mathbf{m}_{\left.2\right|^{\alpha} A}\right\|_{1} .
$$

Let us now use this inequality in equation (24):

$$
\begin{aligned}
\left\|\left(\mathbf{K}_{1}-\mathbf{K}_{2}\right) \mathbf{K}_{3}\right\|_{1} & \leq \sum_{A \subseteq \Omega}\left\|\mathbf{m}_{\left.1\right|^{\alpha} A}-\mathbf{m}_{\left.2\right|^{\alpha} A}\right\|_{1}, \\
\left\|\left(\mathbf{K}_{1}-\mathbf{K}_{2}\right) \mathbf{K}_{3}\right\|_{1} & \leq\left\|\left(\mathbf{K}_{1}-\mathbf{K}_{2}\right)\right\|_{1} .
\end{aligned}
$$

Finally, dividing both sides of the above inequality by $\rho$ gives:

$$
d_{1}\left(m_{1} \odot^{\alpha} m_{3}, m_{2} \odot^{\alpha} m_{3}\right) \leq d_{1}\left(m_{1}, m_{2}\right) .
$$

By definition, this latter inequality means that distance $d_{1}$ is consistent with rule $\odot^{\alpha}$. 
One last result is available for $\alpha$-specialization or $\alpha$-generalization distances defined using the $L^{\infty}$ matrix norm:

Proposition 4. Any $\alpha$-specialization or $\alpha$-generalization distance $d_{\infty}$ defined using the $L^{\infty}$ matrix norm is consistent with its corresponding $\alpha$-junctive combination rule.

Proof. Suppose $m_{1}, m_{2}$ and $m_{3}$ are three mass functions defined on $\Omega . \mathbf{K}_{1}, \mathbf{K}_{2}$ and $\mathbf{K}_{3}$ are their respective evidential matrices with respect to an $\alpha$-junction $\odot^{\alpha}$. The $L^{\infty}$ norm of a matrix is the max of the $L^{\infty}$ norms of its column vectors. Since a column vector of $\mathbf{K}_{i}$ writes as $\mathbf{m}_{\left.i\right|^{\alpha} B}$ with $B \subseteq \Omega$, there exists a subset $X$ such that:

$$
\begin{aligned}
\left\|\left(\mathbf{K}_{1}-\mathbf{K}_{2}\right) \mathbf{K}_{3}\right\|_{\infty} & =\left\|\left(\mathbf{K}_{1}-\mathbf{K}_{2}\right) \mathbf{m}_{\left.3\right|^{\alpha} X}\right\|_{\infty}, \\
& =\left\|\left(\mathbf{K}_{1}-\mathbf{K}_{2}\right) \sum_{Y \subseteq \Omega} m_{\left.3\right|^{\alpha} X}(Y) \mathbf{m}_{Y}\right\|_{\infty}, \\
& \leq \sum_{Y \subseteq \Omega} m_{\left.3\right|^{\alpha} X}(Y)\left\|\left(\mathbf{K}_{1}-\mathbf{K}_{2}\right) \mathbf{m}_{Y}\right\|_{\infty} .
\end{aligned}
$$

Again, the $L^{\infty}$ norm of $\mathbf{K}_{1}-\mathbf{K}_{2}$ is the max of the $L^{\infty}$ norms of its colums vectors $: \max _{Y \subseteq \Omega}\left\|\left(\mathbf{K}_{1}-\mathbf{K}_{2}\right) \mathbf{m}_{Y}\right\|_{\infty}=\left\|\left(\mathbf{K}_{1}-\mathbf{K}_{2}\right)\right\|_{\infty}$. Each term in the previous inequation is maximized by $\left\|\left(\mathbf{K}_{1}-\mathbf{K}_{2}\right)\right\|_{\infty}$ which gives:

$$
\begin{aligned}
\left\|\left(\mathbf{K}_{1}-\mathbf{K}_{2}\right) \mathbf{K}_{3}\right\|_{\infty} & \leq \sum_{Y \subseteq \Omega} m_{\left.3\right|^{\alpha} X}(Y)\left\|\left(\mathbf{K}_{1}-\mathbf{K}_{2}\right)\right\|_{\infty} \\
& \leq\left\|\left(\mathbf{K}_{1}-\mathbf{K}_{2}\right)\right\|_{\infty} .
\end{aligned}
$$

After normalization, the above inequation gives $d_{\infty}\left(m_{1} \odot{ }^{\alpha} m_{3}, m_{2} \odot{ }^{\alpha} m_{3}\right) \leq$ $d_{\infty}\left(m_{1}, m_{2}\right)$.

The following subsection investigates the inconsistency of distances $d_{J}, d_{2}$, $d_{o p 2}$ and $d_{o p \infty}$ with respect to $\alpha$-junctions through a numerical experiment.

\subsection{Experiments on consistency}

This section contains experiments illustrating the (in)consistency of several evidential distances with respect to $\alpha$-conjunctive and $\alpha$-disjunctive rules. For each distance and each value of $\alpha$, one iteration of this experiment consists in picking three random mass functions and check if inequality (20) is verified. The number of times that the property is verified over the number of iterations gives the consistency rate of the distance for a given $\alpha$.

In order to provide such rates, it is necessary to generate mass functions randomly. It is sufficient to draw simple mass functions because cases of inconsistency are more frequent with such functions. Random simple mass functions are drawn uniformly using an algorithm presented in 2. and applied to simple mass function sub-simplices. 
In figure 2, consistency rates for $\alpha$-conjunctive rules and several evidential distances are shown. For this experiment, 1e4 iterations were used. Figure 3 shows the same results for $\alpha$-disjunctive rules.
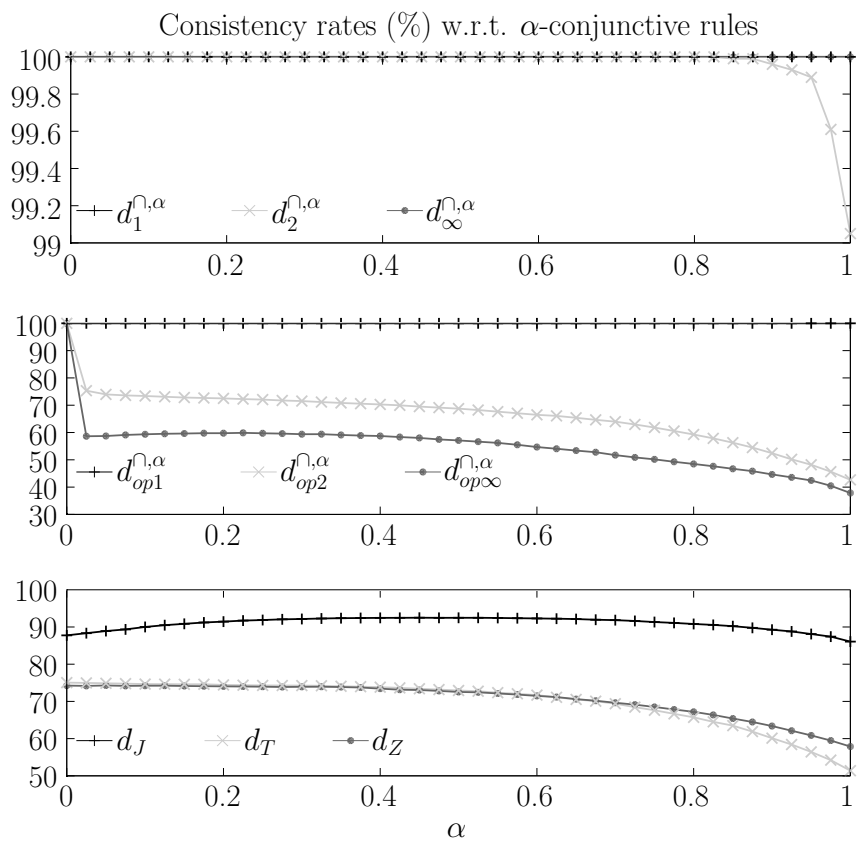

Figure 2: Consistency rates of several evidential distances with $\alpha$-conjunctive rules with respect to parameter $\alpha$.

As expected, the rates of $d_{o p 1}, d_{1}$ and $d_{\infty}$ are $100 \%$ in both the conjunctive and disjunctive cases. A rate under $100 \%$ is sufficient to prove the inconsistency of a distance. We can therefore conclude that $d_{2}, d_{o p 2}$ and $d_{o p \infty}$ are inconsistent when $\alpha \neq 0$. It can be conjectured that they are consistent when $\alpha=0$. The experiment also proves the inconsistency of $d_{J}, d_{T}$ and $d_{Z}$ with any $\alpha$-junction except for the disjunctive case when $\alpha=0$. Their consistency in this latter case may also be conjectured.

\section{Poset structure and evidential matrix-based metrics}

In 17, Jousselme and Maupin shed light on the fact that evidential distances should not process mass functions as if they were any vectors living in the mass space $\mathcal{S}$. Indeed, the power set $2^{\Omega}$ endowed with the inclusion partial order $\subseteq$ is a poset, therefore, $A \subseteq B$ should induce a similar relation between the base vectors $\mathbf{m}_{A}$ and $\mathbf{m}_{B}$ with consequences on distance values.

In [21, we formalized this intuitive principle into the structure property characterizing the existence of an order-isomorphism between an evidential distance 

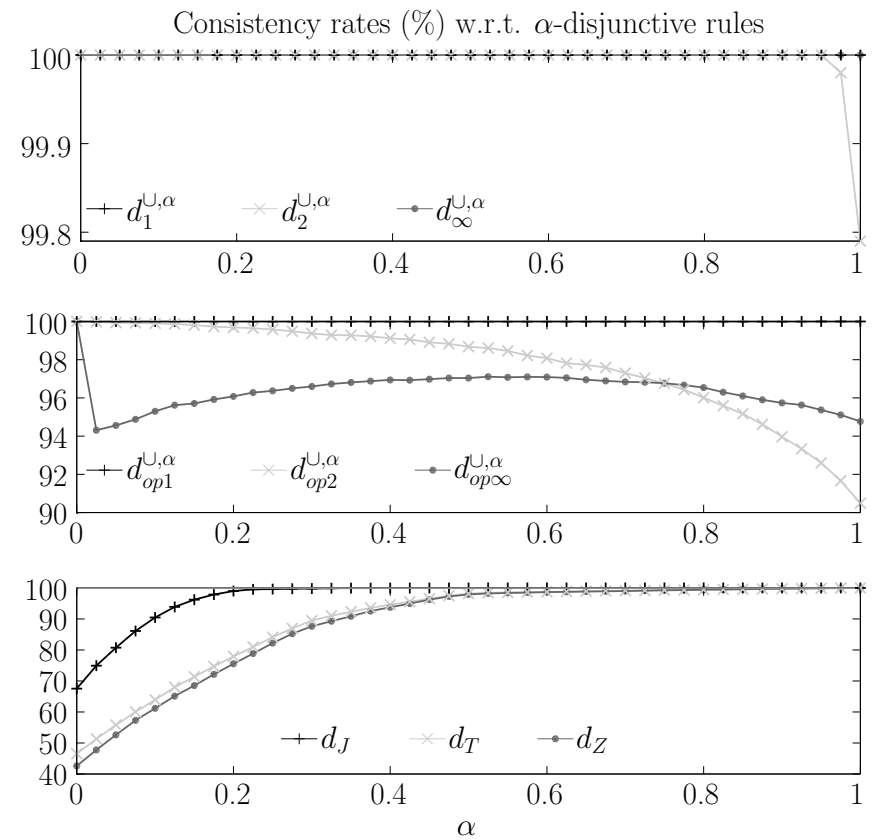

Figure 3: Consistency rates of several evidential distances with $\alpha$-disjunctive rules with respect to parameter $\alpha$.

between categorical mass function and a relevant set distance 3 . Experiments in 21] show that this property is sufficient but not necessary to guarantee that the poset structure is correctly taken into account. In this article, we thus introduce a new property called $\subseteq$-compatibility property:

Definition 6. An evidential distance $d$ is said to be $\subset$-compatible if its restriction to categorical mass functions is not the trivial $\sqrt{4}$ set distance $d_{\text {triv }}$ and if $\forall A B, C \subseteq \Omega$ such that $A \subseteq B \subseteq C$, one has:

$$
d\left(m_{A}, m_{B}\right) \leq d\left(m_{A}, m_{C}\right) .
$$

This property ensures that evidential distances between categorical mass functions have similar dynamics as relevant set distances like the Jaccard distance or the Hamming set distance.

\subsection{Results on the compatibility of matrix-based eviden- tial distances with the inclusion partial order}

Among $\alpha$-specialization or $\alpha$-generalization distances, let us first focus on $d_{o p 1}$ and $d_{\infty}$ :

\footnotetext{
${ }^{3}$ A set distance is a metric on $\left(2^{\Omega}, \subseteq\right)$.

${ }^{4}$ For any sets $A$ and $B$, the trivial metric equals 1 whenever $A \neq B$.
} 
Proposition 5. Let $\Omega$ be a domain and $A, B \subseteq \Omega$. Any $\alpha$-specialization or $\alpha$-generalization distance $d_{o p 1}$ or $d_{\infty}$ defined respectively using the 1-operator or the $L^{\infty}$ matrix norm is $\subseteq$-incompatible. Moreover, for any categorical mass functions $m_{A}$ and $m_{B}$, we have:

$$
d_{o p 1}\left(m_{A}, m_{B}\right)=d_{\infty}\left(m_{A}, m_{B}\right)=d_{\text {triv }}(A, B) .
$$

Proof. Since $d_{o p 1}$ and $d_{\infty}$ are metrics, we have $d_{o p 1}\left(m_{A}, m_{B}\right)=d_{\infty}\left(m_{A}, m_{B}\right)=$ 0 when $A=B$.

Let us now focus on the $A \neq B$ case. From corollary 1 , we know that $d_{o p 1}\left(m_{A}, m_{B}\right)=$ $\frac{1}{\rho}\left\|\mathbf{m}_{A}-\mathbf{m}_{B}\right\|_{1}$. Distances $d_{o p 1}$ and $d_{\infty}$ share the same normalization constant: $\rho=2$. If $A \neq B$, then $\left\|\mathbf{m}_{A}-\mathbf{m}_{B}\right\|_{1}=2$ and thus $d_{o p 1}\left(m_{A}, m_{B}\right)=1$. In addition, for categorical mass functions, we have $\left\|\mathbf{m}_{A}-\mathbf{m}_{B}\right\|_{1}=\left\|\mathbf{m}_{A}-\mathbf{m}_{B}\right\|_{\infty}$, hence:

$$
\begin{aligned}
d_{o p 1}\left(m_{A}, m_{B}\right) & =\frac{1}{2}\left\|\mathbf{m}_{A}-\mathbf{m}_{B}\right\|_{\infty}, \\
\Rightarrow d_{o p 1}\left(m_{A}, m_{B}\right) & \leq \frac{1}{2}\left\|\mathbf{K}_{A}-\mathbf{K}_{B}\right\|_{\infty}, \\
\Leftrightarrow d_{o p 1}\left(m_{A}, m_{B}\right) & \leq d_{\infty}\left(m_{A}, m_{B}\right) .
\end{aligned}
$$

Since $d_{\infty}$ is normalized, we deduce that $d_{\infty}\left(m_{A}, m_{B}\right)=1$.

The above result shows that the definition of the $\subseteq$-compatibility property makes sense because $d_{o p 1}$ is actually equivalent to a vector distance which does not grasp the poset structure of $2^{\Omega}$ at all.

The following lemma shows that the restriction of $d_{1}$ to the set of categorical mass functions is not the trivial distance:

Lemma 4. Suppose $m_{A}$ and $m_{B}$ are two categorical mass functions on $A$ and $B$, two subsets of $\Omega$. Let $d_{1}$ be an $L^{1}$ based distance with respect to a given $\alpha$-junction. $\forall \alpha \in[0,1]$, the following result for $d_{1}$ holds :

$$
d_{1}\left(m_{A}, m_{B}\right)=\frac{2 N}{\rho}\left(1-\frac{\alpha^{\max \{|A \backslash B| ;|B \backslash A|\}}}{2^{|A \Delta B|}}\right) .
$$

The proof of this lemma is found in appendix D, This proof also shows that $\rho=2\left(N-\alpha^{n}\right)$ for $d_{1}$. Equipped with this lemma, one is now able to prove the following proposition:

Proposition 6. Any $\alpha$-specialization or $\alpha$-generalization distance $d_{1}$ defined using the $L^{1}$ matrix norm is $\subseteq$-compatible provided that $\alpha \neq 0$.

Proof. Under the $\alpha=0$ case, we have $d_{1}\left(m_{A} ; m_{B}\right)=d_{\text {triv }}(A, B)$ and $d_{1}$ is $\subseteq$-incompatible.

Let us now suppose that $\alpha>0$. For any sets $A, B \subseteq \Omega$ such that $A \subseteq B$, we have $\max \{|A \backslash B| ;|B \backslash A|\}=|B \backslash A|$ and $|A \Delta B|=|B \backslash A|$, which implies $d_{1}\left(m_{A}, m_{B}\right)=\frac{2 N}{\rho}\left(1-\left(\frac{\alpha}{2}\right)^{|B \backslash A|}\right)$. Now if $C$ is such that $A \subseteq B \subseteq C$, then $|B \backslash A| \leq|C \backslash A|$ which is equivalent to $d_{1}\left(m_{A}, m_{B}\right) \leq d_{1}\left(m_{A}, m_{C}\right)$. 
The next subsection gives more details on the compatibility of other evidential distances with the inclusion partial order thanks to a numerical experiment.

\subsection{Experiments on compatibility with the inclusion par- tial order}

The experiments contained in this section are meant to determine what evidential distances are compliant with inequation (26) when categorical mass functions are compared. To that end, let us study the following toy-example:

Example 4. This example is inspired from an example presented in [16] and re-used in [14, 21]. Let $m_{X}$ and $m_{A_{i}}$ be two categorical mass functions defined on the domain $\Omega=\left\{\omega_{i}\right\}_{i=1}^{n}$, with $X=\left\{\omega_{1}, \omega_{2}, \omega_{3}\right\}$ and $n=|\Omega|=7$. The subset $A_{i}$ varies by successive inclusions of a singleton at each step starting from $\emptyset$ and reaching $\Omega$ at the last computation step:

\begin{tabular}{|c|c|}
\hline$i$ : computation step & focal element $A_{i}$ \\
\hline 0 & $\emptyset$ \\
\hline 1 & $\left\{\omega_{1}\right\}$ \\
\hline 2 & $\left\{\omega_{1}, \omega_{2}\right\}$ \\
\hline 3 & $\left\{\omega_{1}, \omega_{2} \omega_{3}\right\}=X$ \\
\hline 4 & $\left\{\omega_{1}, \ldots, \omega_{4}\right\}$ \\
\hline$\ldots$ & $\ldots$ \\
\hline 7 & $\Omega$ \\
\hline
\end{tabular}

The values of several evidential distances are shown in figure 4 for each step $i$. Note that it is unnecessary to include $\alpha$-generalization distances in this experiment because we know from proposition 1 that they behave similarly as $\alpha$-specialization distances when comparing categorical mass functions.

A $\subseteq$-compatible distance must be decreasing when $i \in\{0 ; \ldots ; 3\}$ and increasing when $i \in\{3 ; \ldots ; 7\}$. To this regard, $d_{2}$ (when $\alpha=0.25$ ), $d_{o p 2}$ (when $\alpha=0.5$ ) and $d_{o p \infty}$ (when $\alpha=0.25$ ) are $\subseteq$-incompatible. It can be conjectured that any matrix-based evidential distance between categorical mass function is the trivial distance when $\alpha=0$.

As for vector distances, $d_{Z}$ is also $\subseteq$-incompatible while it can be proved that $d_{T}$ and $d_{J}$ are both $\subseteq$-compatible.

\section{Meta-information influence on distances}

This section highlights the behavior of $\alpha$-specialization and $\alpha$-generalization distances in the context of a meta-information dependent information fusion problem. We limit ourselves to meta-information items pertaining to $\alpha$-junctions as described in subsection 2.3 i.e. source states.

When the source states are completely unknown, the behavior-based correction introduced by Pichon et al. 25] gives that mass functions delivered by the sources should be replaced with vacuous ones. Any evidential distance is thus null in such a case. Given that the sources are equally poorly informative, this result makes sense. 

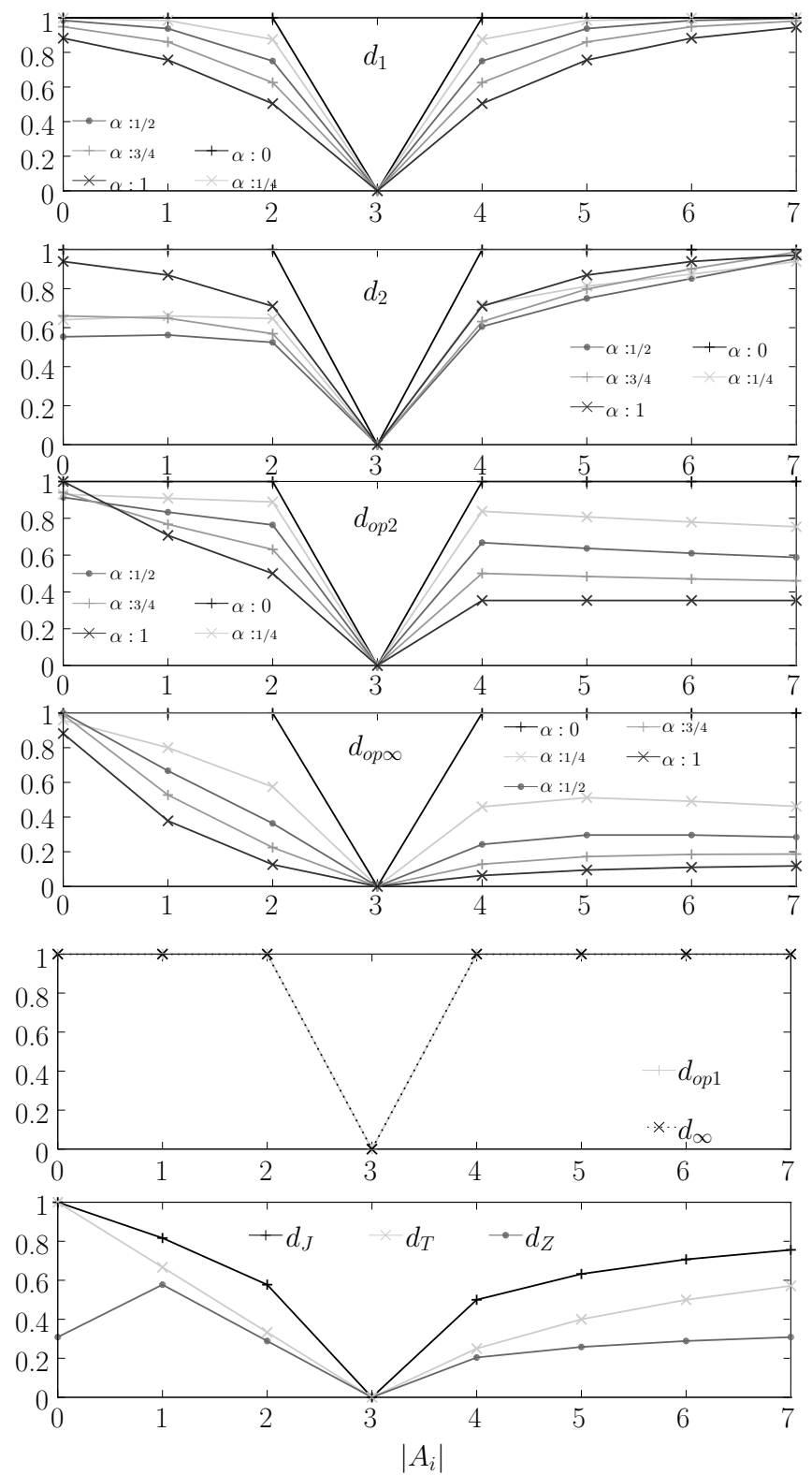

Figure 4: Evidential distances and dissimilarities between two categorical mass functions: $m_{X}$ and $m_{A_{i}}$ defined on $\Omega$, with $|\Omega|=7$ and $|X|=3$. The set $A_{i}$ varies by successive inclusions from $\emptyset$ to $\Omega$ and $A_{3}=X$. The matrix-based evidential distances involved in this experiment are $\alpha$-specialization distances and five values of $\alpha$ are considered: $\left\{0 ; \frac{1}{4} ; \frac{1}{2} ; \frac{3}{4} ; 1\right\}$. 
Let us now examine the opposite situation where meta-information is certain and precise, i.e. the meta-events $\left\{\right.$ source $S_{1}$ is in state $\left.t_{C}^{1}\right\}$ and $\left\{\right.$ source $S_{2}$ is in state $\left.t_{D}^{2}\right\}$ are sure. In this case, the same correction mechanism can be used on each mass function to make them deliver only righteous pieces of evidence. Indeed, back to example 1, we came to the conclusion that a categorical mass function $m_{A}$ delivered by a source in state $t_{C}^{i}$ should be replaced with the function $m \overline{A \Delta C}$. Now, more generally, if $m_{1 \mid t_{C}^{1}}$ denotes the mass function that $S_{1}$ should have delivered, then we deduce:

$$
m_{1 \mid t_{C}^{1}}(Y)=\sum_{\substack{X \subseteq \Omega \\ Y=\bar{C} \Delta X}} m_{1}(X)=m_{1}(\overline{C \Delta Y}),
$$

because $X=\overline{C \Delta Y} \Leftrightarrow Y=\overline{C \Delta X}$.

Using this mechanism, one can compute distances between corrected mass functions without minding meta-information. Although this mechanism is simple and easy to implement, it is however unlikely that such steady meta-information is available in practice.

A more realistic situation is when both sources lie in the same way but one does not known which lie is committed. For instance, many real-world systems possess redundant sensors which may be malfunctioning in the same way because they undergo the same experimental conditions. In this particular situation, a relevant mass function comparison is delivered by some $\alpha$-specialization and $\alpha$-generalization distances in spite of the meta-information imprecision. Indeed, we have the following result:

Proposition 7. Let $m_{1}$ and $m_{2}$ be two mass functions on a domain $\Omega$ delivered by two sources $S_{1}$ and $S_{2}$ that are in states $t_{C}^{1}$ and $t_{C}^{2}$. If $d$ is a specialization distance relying on an $L^{k}$ matrix norm or on the 1-operator norm, then we have:

$$
d\left(m_{1}, m_{2}\right)=d\left(m_{1 \mid t_{C}^{1}}, m_{2 \mid t_{C}^{2}}\right),
$$

with $m_{1 \mid t_{C}^{1}}$ and $m_{2 \mid t_{C}^{2}}$ the mass functions that would have been delivered by $S_{1}$ and $S_{2}$ if they were both fully truthful.

Proof. Let $\Omega$ be a domain and $B, C \subseteq \Omega$. Let $\sigma_{B}$ denote a mapping such that:

$$
\begin{aligned}
\sigma_{B}: 2^{B} & \rightarrow 2^{B}, \\
X & \rightarrow \overline{C \Delta X} \cap B .
\end{aligned}
$$

This mapping is idempotent: $\sigma_{B}^{-1}(Y)=\sigma_{B}(Y)$. Indeed, for any $X \subseteq B$, we 
have:

$$
\begin{aligned}
& \sigma_{B}\left(\sigma_{B}(X)\right)=\overline{(\overline{X \Delta C} \cap B) \Delta C} \cap B, \\
& =((\overline{X \Delta C} \cap B \cap C) \cup \\
& (\overline{\overline{X \Delta C} \cap B} \cap \bar{C})) \cap B, \\
& =(\overline{X \Delta C} \cap B \cap C) \cup \\
& ((X \Delta C) \cup \bar{B}) \cap \bar{C} \cap B, \\
& =(X \cap C \cap B \cap C) \cup \\
& (\bar{X} \cap \bar{C} \cap B \cap C) \cup \\
& (X \Delta C) \cap \bar{C} \cap B \text {, } \\
& =(X \cap C) \cup(X \cap \bar{C} \cup \bar{X} \cap C) \cap \bar{C} \cap B \text {, } \\
& =(X \cap C) \cup(X \cap \bar{C}) \text {, } \\
& =X \text {. }
\end{aligned}
$$

$\sigma_{B}$ is thus a permutation on $2^{B}$.

From equation (30), a corrected mass function $m_{i \mid t_{C}^{i}}$ is obviously equal to $m_{i} \circ \sigma_{\Omega}$. This is sufficient to conclude for the 1-operator norm because this norm is actually a vector norm whose computation is invariant with respect to component permutations. For $L^{k}$ matrix norms, one must first remark that $\forall A, B, X \subseteq \Omega$,

$$
X \cap B=A \cap B \Leftrightarrow \sigma_{\Omega}(X) \cap B=\sigma_{\Omega}(A) \cap B .
$$

It follows that $m_{i \mid B}(A \cap B)=m_{i \mid t_{C}^{i}, B}\left(\sigma_{B}(A)\right)$, with $m_{i \mid t_{C}^{i}, B}=m_{i \mid t_{C}} @ m_{B}$. Let $\mathbf{S}_{i}$ and $\mathbf{S}_{i \mid t_{C}^{i}}$ be the specialization matrices corresponding to $m_{i}$ and $m_{i \mid t_{C}^{i}}$ respectively. Remembering that $\mathbf{m}_{i \mid B}$ and $\mathbf{m}_{i \mid t_{C}^{i}, B}$ are the $j_{B}^{\text {th }}$ (in the binary order sense) column vectors of $\mathbf{S}_{i}$ and $\mathbf{S}_{i \mid t_{C}^{i}}$, it appears that each column of $\mathbf{S}_{i \mid t_{C}^{i}}$ is obtained by permuting non-null elements of the same column in $\mathbf{S}_{i}$. Since $L^{k}$ matrix norm computation is invariant to matrix component permutations, one has :

$$
\begin{aligned}
\left\|\mathbf{S}_{1 \mid t_{C}^{1}}-\mathbf{S}_{2 \mid t_{C}^{2}}\right\|_{k} & =\left\|\mathbf{S}_{1}-\mathbf{S}_{2}\right\|_{k}, \\
\Leftrightarrow d_{k}\left(m_{1}, m_{2}\right) & =d_{k}\left(m_{1 \mid t_{C}^{1}}, m_{2 \mid t_{C}^{2}}\right) .
\end{aligned}
$$

Proposition 7 is interesting in the sense that, when sources are corrupted in same way, the same distance value is obtained as if they were delivering truthful pieces of evidence. This property is illustrated in the following example:

Example 5. The same mass functions as in example 3are used: $m_{1}=m_{X}$ and $m_{2}=0.3 m_{X}+0.5 m_{\bar{X}}+0.2 m_{\Omega}$. However, we chose here $|\Omega|=7$ and $|X|=3$. Suppose $C_{i}$ is a subset varying by successive inclusions from $\emptyset$ to $\Omega$ and $C_{3}=X$. Figure 5 displays distances between $m_{1 \mid t_{C_{i}}^{1}}$ and $m_{2 \mid t_{C_{i}}^{2}}$. 
Evidential distances when sources are both in state $t_{C_{i}}$
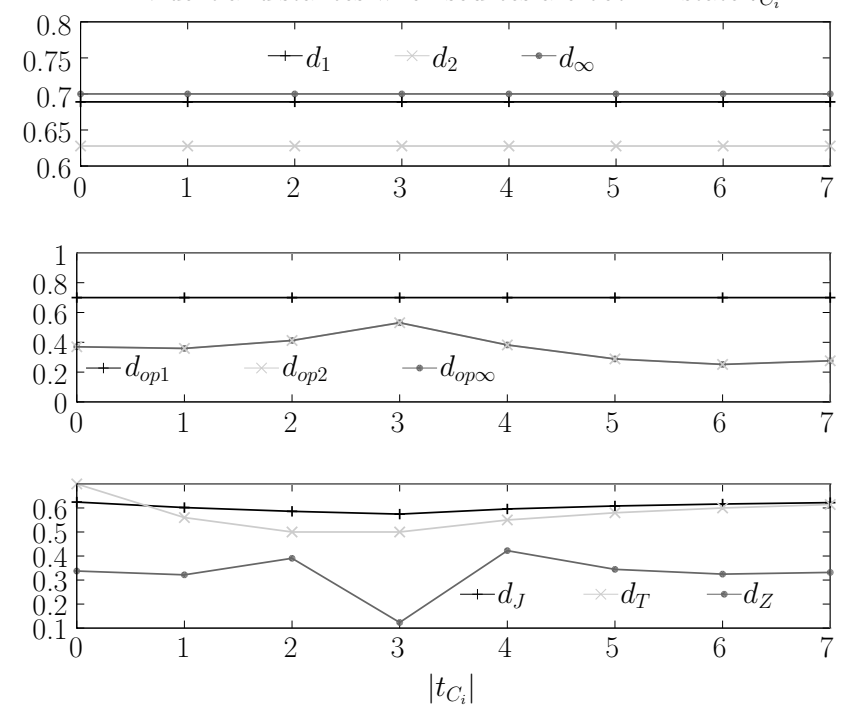

Figure 5: Different evidential distances between same-state-sources. These distances are computed between two given mass functions $m_{1 \mid t_{C_{i}}^{1}}$ and $m_{2 \mid t_{C_{i}}^{2}}$ such that $m_{1}=m_{X}$ and $m_{2}=0.3 m_{X}+0.5 m_{\bar{X}}+0.2 m_{\Omega}$, with $|\Omega|=7$ and $|X|=3$. The state set $C_{i}$ varies by successive inclusions from $\emptyset$ to $\Omega$ and $A_{3}=X$.

Significant discrepancies are observed for vector distances as well as for $d_{o p 2}$ and $d_{o p \infty}$ showing that their distance values are irrelevant if meta-knowledge cannot be filtered.

\section{Conclusion}

In the theory of belief functions, evidence is summarized through a mass function. Since mass functions are in one-to-one correspondence with several matrices, two bodies of evidence can be compared by computing the norm of the difference between such matrices. By investigating several matrix norms, infinite families of evidential distances are introduced in this article. These families generalize the specialization distances presented in [21].

In addition, we prove that some of these distance families are consistent with $\alpha$-junctions in the sense that two mass functions are closer after performing an $\alpha$-junction with the same evidence on both of them. This result is mainly explained by the fact that the definition of matrices used in our evidential distances is closely related to $\alpha$-junctions.

Besides, mass functions are set functions and their domain has a poset structure. It is proved that the sub-family relying on the $L^{1}$ matrix norm is compatible with the inclusion partial order if $\alpha>0$.

Finally, a new aspect of evidential distances is also investigated: the influence 
of meta-information on information source truthfulness. The main conclusion to draw is that when two sources lie in the same way, distances relying on $L^{k}$ matrix norms or on the 1-operator norm are invariant to mass permutations pertaining to the lie if $\alpha=1$.

There are several perspectives for future works. Concerning theoretical aspects, other polytope metrics in other evidential spaces will be investigated along with their potential new properties. In particular, metrics defined in the Belief spact5 are likely to be naturally compatible with the inclusion partial order. Concerning practical aspects, we plan to use the distances introduced in this paper for several mass function approximation problems. Indeed, optimization algorithms are highly dependent on the properties of the metrics on which they rely. Finally, it is also intended to study a system containing redundant sensors. As redundant sensors are supposed to lie in the same way, a distance cited in proposition 7 is a relevant candidate to detect a failure among such sensors.

\section{A Proof of Lemma 2}

Proof. Let $\mathbf{K}_{i, 0}^{\cap}$ and $\mathbf{K}_{i, 0}^{\cup}$ be the 0-specialization matrix and the 0-generalization matrix of $m_{i}$ with $i \in\{1 ; 2\}$. If $\alpha=0$, equation (3) reduces to:

$$
\begin{aligned}
m_{1 \cap^{0} B}(A) & =\sum_{\overline{X \Delta B}=A} m_{1}(X), \\
& =\sum_{X \Delta B=\bar{A}} m_{1}(X), \\
& =m_{1 \cup^{0} B}(\bar{A}) .
\end{aligned}
$$

This is equivalent to $\mathbf{J K}_{i, 0}^{\cap}=\mathbf{K}_{i, 0}^{\cup}$. A similar reasoning as in the proof of proposition 1 gives $d^{\cap, 0}=d^{\cup, 0}$.

Let $\mathbf{K}_{i}^{\bigcap}$ and $\mathbf{K}_{i}^{\cup}$ be the specialization matrix and the generalization matrix of $m_{i}$ with $i \in\{1 ; 2\}$. For any $A$ and $B \subseteq \Omega$ we have:

$$
\begin{aligned}
m_{1 \cup^{1} \bar{B}}(A \cup \bar{B}) & =\sum_{X \cup \bar{B}=A \cup \bar{B}} m_{1}(X), \\
& =\sum_{\bar{X} \cap B=\bar{A} \cap B} m_{1}(X) .
\end{aligned}
$$

Let us observe that $B$ is the disjoint union of $B \backslash X$ with $B \backslash \bar{X}$ as well as the disjoint union of $B \backslash A$ with $B \backslash \bar{A}$. Consequently, $B \backslash \bar{X}=B \backslash \bar{A} \Leftrightarrow B \backslash X=B \backslash A$, which gives:

$$
\begin{aligned}
m_{1 \cup^{1} \bar{B}}(A \cup \bar{B}) & =\sum_{X \cap B=A \cap B} m_{1}(X), \\
& =m_{1 \cap^{1} B}(A \cup B) .
\end{aligned}
$$

\footnotetext{
${ }^{5}$ The Belief space is the subset of $\mathbb{R}^{N}$ in which belief functions live. Mass functions are in bijective correspondence with belief functions via the Möbius transform.
} 
In other words, matrices $\mathbf{K}_{i}^{\cap}$ and $\mathbf{K}_{i}^{\cup}$ contain the same elements but their positions are different. Again, a similar reasoning as in the proof of proposition 1 gives $d^{\cap, 1}=d^{\cup, 1}$.

\section{B Proof of Lemma 3}

Proof. Let $\mathbf{K}_{A}$ denote the evidential matrix of any categorical mass function $m_{A}$ with respect to an $\alpha$-junction. If $A=B$, the properties are trivially proved. If $A \subsetneq B, \exists x \in B$ such that $x \notin A$. Let us now separate the $\alpha$-conjunctive case and the $\alpha$-disjunctive case:

- $\alpha$-conjunction case: Theorem 13 in [27] gives $\mathbf{K}_{A}=\prod_{x^{\prime} \notin A} \mathbf{K}_{\left\{x^{\prime}\right\}}$. This allows us to write:

$\left\|\mathbf{m}_{1 \cap^{\alpha} A}-\mathbf{m}_{2 \cap^{\alpha} A}\right\|_{1}$

$$
\begin{aligned}
& =\left\|\mathbf{K}_{A} \mathbf{m}_{1}-\mathbf{K}_{A} \mathbf{m}_{2}\right\|_{1}, \\
& =\left\|\mathbf{K}_{A}\left(\mathbf{m}_{1}-\mathbf{m}_{2}\right)\right\|_{1}, \\
& =\left\|\mathbf{K}_{\overline{\{x\}}} \mathbf{K}_{A \cup\{x\}}\left(\mathbf{m}_{1}-\mathbf{m}_{2}\right)\right\|_{1} .
\end{aligned}
$$

The very definition of the 1-operator norm for matrix $\mathbf{K}_{\overline{\{x\}}}$ writes as follows:

$$
\left\|\mathbf{K}_{\{x\}}\right\|_{o p 1}=\max _{\mathbf{u} \in \mathbb{R}^{N}} \frac{\left\|\mathbf{K}_{\overline{\{x\}}} \mathbf{u}\right\|_{1}}{\|\mathbf{u}\|_{1}} .
$$

As $\mathbf{K}_{A \cup\{x\}}\left(\mathbf{m}_{1}-\mathbf{m}_{2}\right)$ is a particular vector belonging to $\mathbb{R}^{N}$ and given that $\left\|\mathbf{K}_{\overline{\{x\}}}\right\|_{o p 1}=1$, we have :

$$
1 \geq \frac{\left\|\mathbf{K}_{\{x\}} \mathbf{K}_{A \cup\{x\}}\left(\mathbf{m}_{1}-\mathbf{m}_{2}\right)\right\|_{1}}{\left\|\mathbf{K}_{A \cup\{x\}}\left(\mathbf{m}_{1}-\mathbf{m}_{2}\right)\right\|_{1}} .
$$

Using the above inequality in equation (35) gives:

$$
\left\|\mathbf{m}_{1 \cap^{\alpha} A}-\mathbf{m}_{2 \cap^{\alpha} A}\right\|_{1} \leq\left\|\mathbf{m}_{1 \cap(A \cup\{x\})}-\mathbf{m}_{2 \cap(A \cup\{x\})}\right\|_{1} .
$$

The same result can be used by successive inclusions of any other element $x^{\prime}$ belonging to $B$ while not belonging to $A$, hence:

$$
\left\|\mathbf{m}_{1 \cap^{\alpha} A}-\mathbf{m}_{2 \cap^{\alpha} A}\right\|_{1} \leq\left\|\mathbf{m}_{1 \cap^{\alpha} B}-\mathbf{m}_{2 \cap^{\alpha} B}\right\|_{1} .
$$

- $\alpha$-disjunctive case: Theorem 11 in [27] gives $\mathbf{K}_{A \cup\{x\}}=\prod_{x^{\prime} \in A \cup\{x\}} \mathbf{K}_{\left\{x^{\prime}\right\}}$. This allows us to write:

$$
\begin{aligned}
\left\|\mathbf{m}_{1 \cup^{\alpha}(A \cup\{x\})}-\mathbf{m}_{2 \cup^{\alpha}(A \cup\{x\})}\right\|_{1} \\
=\left\|\mathbf{K}_{A \cup\{x\}} \mathbf{m}_{1}-\mathbf{K}_{A \cup\{x\}} \mathbf{m}_{2}\right\|_{1}, \\
=\left\|\mathbf{K}_{A \cup\{x\}}\left(\mathbf{m}_{1}-\mathbf{m}_{2}\right)\right\|_{1}, \\
=\left\|\mathbf{K}_{\{x\}} \mathbf{K}_{A}\left(\mathbf{m}_{1}-\mathbf{m}_{2}\right)\right\|_{1} .
\end{aligned}
$$


The very definition of the 1-operator norm for matrix $\mathbf{K}_{\{x\}}$ writes as follows:

$$
\left\|\mathbf{K}_{\{x\}}\right\|_{o p 1}=\max _{\mathbf{u} \in \mathbb{R}^{N}} \frac{\left\|\mathbf{K}_{\{x\}} \mathbf{u}\right\|_{1}}{\|\mathbf{u}\|_{1}}
$$

As $\mathbf{K}_{A}\left(\mathbf{m}_{1}-\mathbf{m}_{2}\right)$ is a particular vector belonging to $\mathbb{R}^{N}$ and given that $\left\|\mathbf{K}_{\{x\}}\right\|_{o p 1}=1$, one has :

$$
1 \geq \frac{\left\|\mathbf{K}_{\{x\}} \mathbf{K}_{A}\left(\mathbf{m}_{1}-\mathbf{m}_{2}\right)\right\|_{1}}{\left\|\mathbf{K}_{A}\left(\mathbf{m}_{1}-\mathbf{m}_{2}\right)\right\|_{1}}
$$

Using the above inequality in equation (37) gives:

$$
\left\|\mathbf{m}_{1 \cup^{\alpha}(A \cup\{x\})}-\mathbf{m}_{2 \cup^{\alpha}(A \cup\{x\})}\right\|_{1} \leq\left\|\mathbf{m}_{1 \cup^{\alpha} A}-\mathbf{m}_{2 \cup^{\alpha} A}\right\|_{1} .
$$

The same result can be used by successive inclusions of any other element $x^{\prime}$ belonging to $B$ while not belonging to $A$, hence:

$$
\left\|\mathbf{m}_{1 \cup^{\alpha} A}-\mathbf{m}_{2 \cup^{\alpha} A}\right\|_{1} \geq\left\|\mathbf{m}_{1 \cup^{\alpha} B}-\mathbf{m}_{2 \cup^{\alpha} B}\right\|_{1} .
$$

\section{Proof of corollary 1}

Proof. $\mathbf{K}_{1}$ and $\mathbf{K}_{2}$ are the evidential matrices corresponding to $m_{1}$ and $m_{2}$ with respect to a given $\alpha$-junction. Let us use the following definition of the 1-operator matrix norm for matrix $\mathbf{K}_{1}-\mathbf{K}_{2}$ :

$$
\left\|\mathbf{K}_{1}-\mathbf{K}_{2}\right\|_{o p 1}=\max _{B \subset \Omega} \sum_{A \subset \Omega}\left|\mathbf{K}_{1}(A, B)-\mathbf{K}_{2}(A, B)\right| .
$$

Let us now separate the $\alpha$-conjunctive case and the $\alpha$-disjunctive case:

- In case of an $\alpha$-conjunction, equation (39) also writes:

$$
\left\|\mathbf{K}_{1}-\mathbf{K}_{2}\right\|_{o p 1}=\max _{B \subset \Omega}\left\|\mathbf{m}_{1 \cap{ }^{\alpha} B}-\mathbf{m}_{2 \cap \alpha} B\right\|_{1} .
$$

Using Lemma 3, it is clear that the maximum norm is obtained for the largest set $B$, i.e. when $B=\Omega$, hence:

$$
\begin{aligned}
\left\|\mathbf{K}_{1}-\mathbf{K}_{2}\right\|_{o p 1} & =\left\|\mathbf{m}_{1 \cap \alpha \Omega}-\mathbf{m}_{2 \cap^{\alpha} \Omega}\right\|_{1}, \\
& =\left\|\mathbf{m}_{1}-\mathbf{m}_{2}\right\|_{1} .
\end{aligned}
$$

- In case of an $\alpha$-disjunction, equation (39) writes:

$$
\left\|\mathbf{K}_{1}-\mathbf{K}_{2}\right\|_{o p 1}=\max _{B \subset \Omega}\left\|\mathbf{m}_{1 \cup^{\alpha} B}-\mathbf{m}_{2 \cup^{\alpha} B}\right\|_{1} .
$$


Using Lemma 3, it is clear that the maximum norm is obtained for the smallest set $B$, i.e. when $B=\emptyset$, hence:

$$
\begin{aligned}
\left\|\mathbf{K}_{1}-\mathbf{K}_{2}\right\|_{o p 1} & =\left\|\mathbf{m}_{1 \cup^{\alpha} \emptyset}-\mathbf{m}_{2 \cup^{\alpha} \emptyset}\right\|_{1}, \\
& =\left\|\mathbf{m}_{1}-\mathbf{m}_{2}\right\|_{1} .
\end{aligned}
$$

\section{Proof of Lemma 4}

Proof. Let us first consider that $d_{1}$ is defined with respect to an $\alpha$-conjunctive rule. Let $\mathbf{K}_{A}$ and $\mathbf{K}_{B}$ be the evidential matrices corresponding to $m_{A}$ and $m_{B}$ with respect to the rule $\AA^{\alpha}$ and let $\rho$ be a normalization coefficient. By definition of distance $d_{1}$, one can write:

$$
\begin{aligned}
d_{1}\left(m_{A}, m_{B}\right) & =\frac{1}{\rho}\left\|\mathbf{K}_{A}-\mathbf{K}_{B}\right\|_{1}, \\
& =\frac{1}{\rho} \sum_{X, Y}\left|K_{A}(X, Y)-K_{B}(X, Y)\right| .
\end{aligned}
$$

Given results in [18, it is known that the element $K_{A}(X, Y)$ of matrix $\mathbf{K}_{A}$ is non-null if and only if $A \cap Y \subseteq X \subseteq \overline{A \Delta Y}$. Consequently, matrix elements are actually subtracted if $A \cap Y \subseteq X \subseteq \overline{A \Delta Y}$ and $B \cap Y \subseteq X \subseteq \overline{B \Delta Y}$. Let us analyze this situation:

- If $X$ is a superset of both $A \cap Y$ and $B \cap Y$, then $X$ is a superset of $(A \cup B) \cap Y=(A \cap Y) \cup(B \cap Y)$.

- If $X$ is a subset of both $\overline{A \Delta Y}$ and $\overline{B \Delta Y}$, then it is a subset of $\overline{A \cup B \cup Y} \cup$ $(A \cap B \cap Y)=\overline{A \Delta Y} \cap \overline{B \Delta Y}$.

Let $I=A \cap B \cap Y$ and $U=A \cup B \cup Y$. One can write:

$$
\begin{aligned}
& \left\|\mathbf{K}_{A}-\mathbf{K}_{B}\right\|_{1}=\sum_{\substack{X, Y \\
(A \cup B) \cap Y \subseteq X \subseteq \bar{U} \cup I}}\left|K_{A}(X, Y)-K_{B}(X, Y)\right| \\
& +\sum_{\substack{X, Y \\
A \cap Y \subseteq X \subseteq \overline{A \Delta Y} \\
B \cap Y \nsubseteq X \text { or } X \nsubseteq \overline{B \Delta Y}}} K_{A}(X, Y)+\sum_{\substack{X, Y \\
B \cap Y \subseteq X \subseteq \overline{B \Delta Y} \\
A \cap Y \nsubseteq X \text { or } X \nsubseteq \overline{A \Delta Y}}} K_{B}(X, Y), \\
& =\left\|\mathbf{K}_{A}\right\|_{1}+\left\|\mathbf{K}_{B}\right\|_{1}+\sum_{\substack{X, Y \\
(A \cup B) Y Y \subseteq \\
X \subseteq \bar{U} \cup I}} G(X, Y),
\end{aligned}
$$

with $\mathbf{G}$ a matrix whose elements are such that $G(X, Y)=\left|K_{A}(X, Y)-K_{B}(X, Y)\right|-$ $K_{A}(X, Y)-K_{B}(X, Y)$. Given that the $L^{1}$ norm of any evidential matrix is $N$ 
and that evidential matrices are positive, we can also write:

$$
\left\|\mathbf{K}_{A}-\mathbf{K}_{B}\right\|_{1}=2 N-2 \sum_{\substack{X, Y \\(A \cup B) \cap Y \subseteq X \\ X \subseteq U \cup I}} K_{A \wedge B}(X, Y),
$$

with $\mathbf{K}_{A \wedge B}$ the entry-wise minimum of matrices $\mathbf{K}_{A}$ and $\mathbf{K}_{B}$. It can be proved that $(A \cup B) \cap Y \subseteq \bar{U} \cup I \Leftrightarrow Y \subseteq \overline{A \Delta B}$, which gives:

$$
\left\|\mathbf{K}_{A}-\mathbf{K}_{B}\right\|_{1}=2 N-2 \sum_{\substack{Y \\ Y \subseteq \bar{A} \Delta B}} \sum_{\substack{X \\(A \cup B) \cap Y \subseteq X \\ X \subseteq \bar{U} \cup I}} K_{A \wedge B}(X, Y) .
$$

Given that $Y \subseteq \overline{A \Delta B}$, we have $(A \cup B) \cap Y=A \cap B \cap Y$. Consequently, it appears that all subsets $X$ are the union of $I$ with a given subset of $\bar{U}$. The second sum can thus be re-indexed as follows:

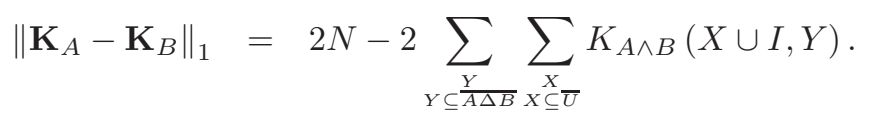

From proposition 1 in [18, we have that for any set $C$ such that $C \cap X=\emptyset$ : $K_{A \wedge B}(X \cup C, Y)$

$$
\begin{aligned}
& =\min \left\{\frac{\alpha^{|\overline{A \Delta Y}|}}{\bar{\alpha}^{|A \cap Y|}}\left(\frac{\bar{\alpha}}{\alpha}\right)^{|X \cup C|} ; \frac{\alpha^{|\overline{B \Delta Y}|}}{\bar{\alpha}^{|B \cap Y|}}\left(\frac{\bar{\alpha}}{\alpha}\right)^{|X \cup C|}\right\}, \\
& =\min \left\{\frac{\alpha^{|\overline{A \Delta Y}|}}{\bar{\alpha}^{|A \cap Y|}} ; \frac{\alpha^{|\overline{B \Delta Y}|}}{\bar{\alpha}^{|B \cap Y|}}\right\}\left(\frac{\bar{\alpha}}{\alpha}\right)^{|X \cup C|}, \\
& =\min \left\{\frac{\alpha^{|\overline{A \Delta Y}|}}{\bar{\alpha}^{|A \cap Y|}} ; \frac{\alpha^{|\overline{B \Delta Y}|}}{\bar{\alpha}^{|B \cap Y|}}\right\}\left(\frac{\bar{\alpha}}{\alpha}\right)^{|X|+|C|} .
\end{aligned}
$$

The sum over subsets $X$ can thus be explicitly computed:

$$
\begin{aligned}
\sum_{\substack{X \\
X \subseteq \bar{U}}} K_{A \wedge B}(X \cup I, Y) & \\
= & \min \left\{\frac{\alpha^{|\overline{A \Delta Y}|}}{\bar{\alpha}^{|A \cap Y|}} ; \frac{\alpha^{|\overline{B \Delta Y}|}}{\bar{\alpha}^{|B \cap Y|}}\right\}\left(\frac{\bar{\alpha}}{\alpha}\right)^{|I|} \sum_{\substack{X \\
x \subseteq \bar{U}}}\left(\frac{\bar{\alpha}}{\alpha}\right)^{|X|}, \\
& =\min \left\{\frac{\alpha^{|\overline{A \Delta Y}|}}{\bar{\alpha}^{|A \cap Y|}} ; \frac{\alpha^{|\overline{B \Delta Y}|}}{\bar{\alpha}^{|B \cap Y|}}\right\}\left(\frac{\bar{\alpha}}{\alpha}\right)^{|I|} \sum_{i=1}^{|\bar{U}|}\left(\begin{array}{c}
|\bar{U}| \\
i
\end{array}\right)\left(\frac{\bar{\alpha}}{\alpha}\right)^{i}, \\
& =\min \left\{\frac{\alpha^{|\overline{A \Delta Y}|}}{\bar{\alpha}^{|A \cap Y|}} ; \frac{\alpha^{|\overline{B \Delta Y}|}}{\bar{\alpha}^{|B \cap Y|}}\right\}\left(\frac{\bar{\alpha}}{\alpha}\right)^{|I|}\left(\frac{\bar{\alpha}}{\alpha}+1\right)^{|\bar{U}|}, \\
& =\min \left\{\frac{\alpha^{|\overline{A \Delta Y}|}}{\bar{\alpha}^{|A \cap Y|}} ; \frac{\alpha^{|\overline{B \Delta Y}|}}{\bar{\alpha}^{|B \cap Y|}}\right\}\left(\frac{\bar{\alpha}}{\alpha}\right)^{|I|} \alpha^{-|\bar{U}|} .
\end{aligned}
$$


Besides, since $Y \subseteq \overline{A \triangle B}, Y$ is the union of two disjoint subsets $W \subseteq A \cap B$ and $Z \subseteq \overline{A \cup B}$. In particular, we have:

- $|\overline{A \Delta Y}|=|\overline{A \Delta(W \cup Z)}|=|\overline{(A \cup Z) \backslash W}|=n-|A|-|Z|+|W|$ and likewise $|\overline{B \Delta Y}|=n-|B|-|Z|+|W|$

- $|A \cap Y|=|A \cap(W \cup Z)|=|W|$ and likewise $|B \cap Y|=|W|$,

- $|I|=|W|$,

- $|\bar{U}|=|\overline{A \cup B \cup Z}|=n-|A \cup B|+|Z|$.

This decomposition of $Y$ allows us to write:

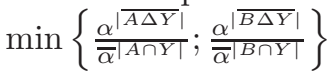

$$
\begin{aligned}
& =\min \left\{\frac{\alpha^{n-|A|-|Z|+|W|}}{\bar{\alpha}^{|W|}} ; \frac{\alpha^{n-|B|-|Z|+|W|}}{\bar{\alpha}^{|W|}}\right\}, \\
& =\min \left\{\alpha^{-|A|} ; \alpha^{-|B|}\right\} \frac{\alpha^{n-|Z|+|W|}}{\bar{\alpha}^{|W|}}, \\
& =\frac{\alpha^{n-|Z|+|W|-\min \{|A| ;|B|\}}}{\bar{\alpha}^{|W|}} .
\end{aligned}
$$

Going back to the computation of $\left\|\mathbf{K}_{A}-\mathbf{K}_{B}\right\|_{1}$, simplifications for the terms at the power of $|W|$ and $|Z|$ are observed, which gives:

$\left\|\mathbf{K}_{A}-\mathbf{K}_{B}\right\|_{1}$

$$
\begin{aligned}
& =2 N-2 \sum_{\substack{W \\
W \subseteq A \cap B}} \sum_{\substack{Z \\
\bigcup^{Z} \cup \cup B}} \alpha^{|A \cup B|-\min \{|A| ;|B|\}}, \\
& =2 N-2 \alpha^{|A \cup B|-\min \{|A| ;|B|\}} 2^{|A \cap B|} 2^{|\overline{A \cup B}|}, \\
& =2 N\left(1-\alpha^{|A \cup B|-\min \{|A| ;|B|\}} 2^{|A \cap B|-|A \cup B|}\right), \\
& =2 N\left(1-\frac{\alpha^{\max \{|A \backslash B| ;|B \backslash A|\}}}{2^{|A \Delta B|}}\right) .
\end{aligned}
$$

Let us now investigate the $\alpha$-disjunctive case. For the remainder of this proof, we thus distinguish $d_{1, \cap}$ and $d_{1, \cup}$. From proposition 1, one can write:

$$
\begin{aligned}
d_{1, \cup}\left(m_{A}, m_{B}\right) & =d_{1, \cap}\left(\bar{m}_{A}, \bar{m}_{B}\right), \\
& =d_{1, \cap}\left(m_{\bar{A}}, m_{\bar{B}}\right), \\
& =\frac{2 N}{\rho}\left(1-\frac{\alpha^{\max \{|\bar{A} \backslash \bar{B}| ;|\bar{B} \backslash \bar{A}|\}}}{\left.2^{|\bar{A} \Delta \bar{B}|}\right),}\right. \\
& =\frac{2 N}{\rho}\left(1-\frac{\alpha^{\max \{|B \backslash A| ;|A \backslash B|\}}}{2^{|A \Delta B|}}\right), \\
& =d_{1, \cap}\left(m_{A}, m_{B}\right) .
\end{aligned}
$$




\section{Acknowledgment}

The authors are indebted to the anonymous reviewers for their valuable comments and suggestions to improve the quality of the paper and to pave the way for future works.

\section{References}

[1] Mathias Bauer. Approximation algorithms and decision making in the Dempster-Shafer theory of evidence an empirical study. International Journal of Approximate Reasoning, 17(23):217 - 237, 1997.

[2] Thomas Burger and Sebastien Destercke. How to randomly generate mass functions. International Journal of Uncertainty, Fuzziness and KnowledgeBased Systems, pages 645-673, 2013.

[3] F. Cuzzolin. Visions of a generalized probability theory. Lambert Academic Publishing, London, UK, 2014.

[4] Fabio Cuzzolin. Geometry of Dempster's rule of combination. IEEE Transactions on Systems, Man, and Cybernetics. Part B: Cybernetics, 34(2):961 $-977,2004$.

[5] Fabio Cuzzolin. Two new bayesian approximations of belief functions based on convex geometry. IEEE Transactions on Systems, Man, and Cybernetics, Part B: Cybernetics, Volume 37(4):993-1008, 2007.

[6] Fabio Cuzzolin. A geometric approach to the theory of evidence. IEEE Transactions on Systems, Man and Cybernetics - Part C: Application And Reviews, 38(4):522 - 534, 2008.

[7] Fabio Cuzzolin. Lp consonant approximation of belief functions in the mass space. In Proceedings of the 7th International Symposium on Imprecise Probability: Theories and Applications, pages 1 - 10, Innsbruck, Austria., 2011.

[8] Fabio Cuzzolin. On consistent approximations of belief functions in the mass space. In Weiru Liu, editor, Symbolic and Quantitative Approaches to Reasoning with Uncertainty, volume 6717 of Lecture Notes in Computer Science, pages 287-298. Springer Berlin Heidelberg, 2011.

[9] A.P. Dempster. Upper and lower probabilities induced by a multiple valued mapping. Annals of Mathematical Statistics, 38(2):325 - 339, 1967.

[10] Javier. Diaz, Maria. Rifqi, and Bernadette. Bouchon-Meunier. A similarity measure between basic belief assignments. In Proceedings of the 9th International Conference on Information Fusion (FUSION 2006), pages 1-6, Florence, Italy, 2006. 
[11] Zied Elouedi, Khaled Mellouli, and Philippe Smets. Assessing sensor reliability for multisensor data fusion within the transferable belief model. IEEE Transactions on Systems, Man, and Cybernetics. Part B: Cybernetics, 34(1):782 - 787, 2004.

[12] Dale Fixen and Ronald Mahler. The modified Dempster-Shafer approach to classification. IEEE Transactions on Systems, Man and Cybernetics. Part A: Systems and humans, 27(1):96 - 104, 1997.

[13] Maurice Fréchet. Sur quelques points du calcul fonctionnel. PhD thesis, Faculté des Sciences de Paris, 1906.

[14] Deqiang Han, Jean Dezert, Chongzhao Han, and Yi Yang. New dissimilarity measure in evidence theory. In Proceedings of the 14th International Conference on Information Fusion (FUSION 2011), pages 1 - 7, Chicago, IL. United States, 2011.

[15] David Harmanec. Faithful approximations of belief functions. In K.B. Laskey and H. Prade, editors, Uncertainty in Artificial Intelligence (UAI99) 15, Stockholm (Sweden), 1999.

[16] Anne-Laure Jousselme, Dominic Grenier, and Eloi Bossé. A new distance between two bodies of evidence. Information Fusion, 2:91-101, 2001.

[17] Anne-Laure Jousselme and Patrick Maupin. Distances in evidence theory : Comprehensive survey and generalizations. International Journal of Approximate Reasoning, 53:118 - 145, 2012.

[18] John Klein, Mehena Loudahi, Jean-Marc Vannobel, and Olivier Colot. $\alpha$ junctions of categorical mass functions. In Fabio Cuzzolin, editor, Belief Functions: Theory and Applications, volume 8764 of Lecture Notes in Artificial Intelligence, pages 1-10. Springer International Publishing, 2014.

[19] George J. Klir and Mark J. Wierman. Uncertainty-Based Information: Elements of Generalized Information Theory,. Springer, 1999.

[20] Weiru Liu. Analysing the degree of conflict among belief functions. Artificial Intelligence, 170(11):909-924, 2006.

[21] Mehena Loudahi, John Klein, Jean-Marc Vannobel, and Olivier Colot. New distances between bodies of evidence based on dempsterian specialization matrices and their consistency with the conjunctive combination rule. International Journal of Approximate Reasoning, 55(5):1093 - 1112, 2014.

[22] W.L. Perry and H.E. Stephanou. Belief function divergence as a classifier. In Intelligent Control, 1991., Proceedings of the 1991 IEEE International Symposium on, pages 280-285, Aug 1991. 
[23] Frédéric Pichon. On the $\alpha$-conjunctions for combining belief functions. In Thierry Denoeux and Marie-Hlne Masson, editors, Belief Functions: Theory and Applications, volume 164 of Advances in Intelligent and Soft Computing, pages 285-292. Springer Berlin Heidelberg, 2012.

[24] Frédéric Pichon and Thierry Denœux. Interpretation and computation of $\alpha$-junctions for combining belief functions. In 6th International Symposium on Imprecise Probability: Theories and Applications ISIPTA-09, pages 1 10, Durham, United Kingdom, July 2009.

[25] Frédéric Pichon, Didier Dubois, and Thierry Denœux. Relevance and truthfulness in information correction and fusion. International Journal of Approximate Reasoning, 53(2):159 - 175, 2012. Theory of Belief Functions (BELIEF 2010).

[26] Glenn Shafer. A mathematical theory of evidence. Princeton University Press, 1976.

[27] P. Smets. The alpha-junctions: Combination operators applicable to belief functions. In First Int. Joint Conference on Qualitative and Quantitative Practical Reasoning (ECSQUARU-FAPR'97), volume 1244 of Lecture Notes in Computer Sciences, pages 131-153. Springer International Publishing, Bad Honef, Germany, 1997.

[28] Philippe Smets. The application of the matrix calculus to belief functions. International Journal of Approximate Reasoning, 31:1-30, 2002.

[29] Philippe Smets and Robert Kennes. The transferable belief model. Artificial Intelligence, 66(2):191 - 234, 1994.

[30] H.E. Stephanou and S.-Y. Lu. Measuring consensus effectiveness by a generalized entropy criterion. Pattern Analysis and Machine Intelligence, IEEE Transactions on, 10(4):544-554, Jul 1988.

[31] Zachary Sunberg and Jonathan Rogers. A belief function distance metric for orderable sets. Information Fusion, 14:361 - 373, 2013.

[32] Bjornar Tessem. Approximations for efficient computation in the theory of evidence. Artificial Intelligence, 61:315 - 329, 1993.

[33] Thomas Weiler and Ulrich Bodenhofer. Approximation of belief functions by minimizing euclidean distances. International Journal of Uncertainty, Fuzziness and Knowledge-Based Systems, 11(06):170 -177, 2003.

[34] Lalla Mariem Zouhal and Thierry Denœux. An evidence-theoretic k-nn rule with parameter optimisation. IEEE Transactions on Systems, Man and Cybernetics. Part C: Application and reviews, 28(2):263-271, 1998. 